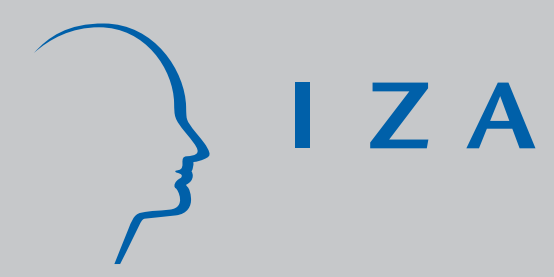

IZA DP No. 2319

Analyzing Cost Efficient Production Behavior Under Economies of Scope:

A Nonparametric Methodology

Laurens Cherchye

Bram De Rock

Frederic Vermeulen

September 2006 


\title{
Analyzing Cost Efficient Production Behavior Under Economies of Scope: A Nonparametric Methodology
}

\author{
Laurens Cherchye \\ University of Leuven and FWO-Vlaanderen \\ Bram De Rock \\ University of Leuven \\ Frederic Vermeulen \\ Tilburg University, CentER, Netspar \\ and IZA Bonn
}

Discussion Paper No. 2319

September 2006

\author{
IZA \\ P.O. Box 7240 \\ 53072 Bonn \\ Germany \\ Phone: +49-228-3894-0 \\ Fax: +49-228-3894-180 \\ E-mail: iza@iza.org
}

\begin{abstract}
Any opinions expressed here are those of the author(s) and not those of the institute. Research disseminated by IZA may include views on policy, but the institute itself takes no institutional policy positions.

The Institute for the Study of Labor (IZA) in Bonn is a local and virtual international research center and a place of communication between science, politics and business. IZA is an independent nonprofit company supported by Deutsche Post World Net. The center is associated with the University of Bonn and offers a stimulating research environment through its research networks, research support, and visitors and doctoral programs. IZA engages in (i) original and internationally competitive research in all fields of labor economics, (ii) development of policy concepts, and (iii) dissemination of research results and concepts to the interested public.
\end{abstract}

IZA Discussion Papers often represent preliminary work and are circulated to encourage discussion. Citation of such a paper should account for its provisional character. A revised version may be available directly from the author. 


\section{ABSTRACT \\ Analyzing Cost Efficient Production Behavior Under Economies of Scope: A Nonparametric Methodology* \\ In designing a production model for firms that generate multiple outputs, we take as a starting point that such multi-output production refers to economies of scope, which in turn originate from joint input use and input externalities. We provide a nonparametric characterization of cost efficient behavior under these conditions, and subsequently institute necessary and sufficient conditions for data consistency with such efficient behavior that only include observed firm demand and supply data. We illustrate our methodology by examining the cost efficiency of research programs in Economics and Business Management faculties of Dutch universities. This application shows that the proposed methodology may entail robust conclusions regarding cost efficiency differences between universities within specific specialization areas, even when using shadow prices to evaluate the different inputs.}

JEL Classification: C12, C14, D21, P32, Q12

Keywords: production behavior, multi-product firms, input externalities, joint input use, economies of scope, nonparametric tests

Corresponding author:

Frederic Vermeulen

Tilburg University

P.O. Box 90153

NL-5000 LE Tilburg

The Netherlands

E-mail: frederic.vermeulen@uvt.nl

\footnotetext{
*We thank two anonymous referees for insightful comments and suggestions, which substantially improved the paper.
} 


\section{Introduction}

We present a novel approach for analyzing the cost efficiency of multi-output firms. Our starting point is that such multi-output production is essentially induced by the presence of economies of scope, which may loosely be defined as "situations where the average total cost of production decreases as a result of increasing the number of different goods produced" (see, e.g., Baumol et al., 1982). Given this, we stress that we do not want to present a methodology for investigating the extent to which economies of scope are actually present. Rather, as we explain below, we present a toolkit for analyzing cost efficient production behavior that exploits a number of specific features related to scope economies. This is an important difference between our approach and most other contributions on scope economies in the production literature, which indeed essentially aim at recovering whether and to what extent the production technology under study is characterized by economies of scope. See, e.g., Kim et al. (2005) and references therein.

A first specificity of our approach is that it is embedded in a nonparametric methodology. Such an approach has some well-known advantages when compared to a parametric approach. ${ }^{1}$ For example, it does not rely on a functional specification of a firm's production technology. In fact, economic theory generally does not imply a particular functional form for the production technology, and reliable empirical specification tests are not available in many cases. As a result, rejections of restrictions imposed by economic theory may be due to an illspecified functional form and do not necessarily reject the theory as such. Yatchew (1998, 669-670), for instance, cites this lack of theoretically superior functional (parametric) specifications as a prime motivation for using nonparametric analytical tools. Generally, within a parametric framework it is possible to test production properties conditional upon some a priori postulated functional form for representing the production technology, but the functional form as such is usually not testable. Furthermore, the nonparametric methodology deals in a very natural way with the widely observed simultaneous occurrence of multiple inputs and outputs. Finally, it easily accounts for the possibility that input-output combinations do not necessarily have to lie on the production frontier: production behavior can be analyzed while allowing for observed inefficiencies. ${ }^{2}$

As for our specific methodology, rather than resorting to some parametric specification of the production technology, we use the mere technological postulates of nested input requirement sets (or free output disposability) and convexity in output space. Both technology properties have often been used in a nonparametric setting. For example, Varian (1984) suggests the assumption of nested input sets, while Petersen (1990) and Bogetoft (1996) suggest the use of convexity in output space. As we will discuss, these minimal assumptions allow for analyzing cost efficient behavior from the raw price and quantity data by exploiting specific features of production processes characterized by economies of scope.

The second specificity of our approach then pertains to this particular (scope economies) interpretation of our empirical cost efficiency conditions. More specifically, we take it that the very nature of scope economies lies in joint input use and input externalities. The cost rationalizing effect of joint input use for multi-output firms is evident. For instance, as for our own empirical application, senior researchers can serve as an input in the production of both

\footnotetext{
${ }^{1}$ See, e.g., Varian (1984), Färe et al. (1994) and Cooper et al. (2000) for introductory texts on nonparametric production and efficiency analysis.

2 See, e.g., the "subset rationalization" concept of Banker and Maindiratta (1988) and the "goodness-of-fit" concept of Varian (1990), which essentially reconcile the neoclassical nonparametric production analysis literature (see, e.g., Afriat, 1972; Hanoch and Rothschild, 1972; and Varian, 1984) and the Data Envelopment Analysis (DEA) literature. [The term DEA, which was introduced by Charnes et al. (1978), is often used for summarizing the literature on nonparametric efficiency analysis.]
} 
academic publications and doctoral dissertations that are delivered by the research production unit. Within the same setting, input externalities occur when the presence of a distinguished scholar has beneficial effects on the productivity of other members of the research unit, even if she or he is not directly involved in the production of the associated research output. More generally, input externalities refer to cost saving (or productivity enhancing) effects to be attributed to inputs (employed by the same production unit) that are not used in a direct manner for the production of the output under consideration.

While the illustrative application in the current paper pertains to the specific case of academic research production, it is worth stressing that scope economies prevail in a wide variety of real-life situations, in the public sector (e.g. public railway companies that simultaneously provide freight and passenger transport) as well as in the private sector (e.g. banks that also provide insurance services). More generally, given our starting position that scope economies (originating from jointly used inputs and input externalities) form the very economic motivation for multi-output production, we believe that our methodology becomes a useful analytical tool as soon as the production behavior is characterized by multiple outputs.

Finally, our method does not a priori impose economies of scope; it does not assume any structure regarding the nature of the effects resulting from joint input use and input externalities. In addition, it does not presume that the empirical analyst knows which (parts of the) observed input quantities represent joint use or are attributed to specific outputs. Indeed, such non-observability of the input distribution is often the case in real-life applications. That is, although we can observe aggregate inputs (for example, the numbers of senior and junior researchers), it may be quite difficult to determine which inputs are directly associated with what output (academic publications or doctoral dissertations). In the concluding section, we indicate how the presented model can be refined in the case that such additional information regarding the input quantity distribution is available.

Still, even though we impose minimal a priori structure regarding the nature of the scope economies or the input distribution, we can derive testable conditions for cost efficient behavior. Interestingly, these conditions are expressed in terms of observable (aggregate) price and input-output quantity information. [In fact, we also extend our tools for nonparametric cost efficiency analysis to apply when only input-output quantity and no price information is available.] That is, there is no need to disaggregate the observed firm demand and supply data to analyze the firm's cost efficiency, which make our conditions easy to implement in practice.

To demonstrate its practical usefulness, we apply our methodology by assessing the cost efficiency of research programs in Economics and Business Management Faculties of Dutch universities. Our data cover the period 1996-2000 and were delivered by the universities in the context of the quinquennial assessment of university research conducted under the auspices of the Association of Dutch Universities (VSNU). As argued above, the multi-output research production is likely to be characterized by economies of scope, which makes this data set well fit to illustrate our methodology.

The rest of the paper is organized as follows. Section 2 presents a nonparametric characterization of cost efficient production behavior under economies of scope. As we will discuss, this provides nonparametric necessary and sufficient conditions for efficient behavior that are expressed in terms of unobservable price and quantity information. Section 3 subsequently presents the corresponding necessary and sufficient cost efficiency conditions that solely use observable information, and which are easy to implement in practice. Section 4 presents our empirical application to research programs in Dutch Economics and Business Management Faculties. Section 5 summarizes and provides some concluding remarks 
regarding potential extensions and refinements of the presented methodology. The appendix contains the proofs of our results.

\section{Cost efficient production behavior under economies of scope: a nonparametric characterization}

We consider firms (broadly defined) that use an $m$-valued input vector $\mathbf{x} \in \Re_{+}^{m}$ to produce an $s$-valued output vector $\mathbf{y} \in \mathfrak{R}_{+}^{s}$; in the following, we let $K=\{1, \ldots, s\}$ denote the output index set. Next, we assume a data set with $t$ firm observations; we use $S=\{1, \ldots, t\}$ to denote the corresponding index set. For each observation $i \in S$, we observe the output vector $\mathbf{y}^{i}$, the corresponding input vector $\mathbf{x}^{i}$ and the input price vector $\mathbf{p}^{i} \in \mathfrak{R}_{++}^{m}$. [In a following step, we relax the assumption that input prices are observed.]

In what follows, we consider the most general production processes, which correspond to our interpretation of scope economies discussed in the Introduction. Specifically, we take account of the fact that the production process of each output may be characterized by production externalities and joint input use. To do so, we define decomposed input vectors $\hat{\mathbf{x}}=\left(\begin{array}{llll}\mathbf{x}_{1}^{\prime} & \ldots & \mathbf{x}_{s}^{\prime} & \mathbf{x}_{s+1}^{\prime}\end{array}\right)^{\prime}$ for an (aggregate) input vector $\mathbf{x}$ such that

$$
\mathbf{x}=\mathbf{x}_{1}+\ldots+\mathbf{x}_{s}+\mathbf{x}_{s+1} \text { and } \mathbf{0} \leq \mathbf{x}_{l} \leq \mathbf{x} \text { for } l=1, \ldots, s+1
$$

In this specification, each component $\mathbf{x}_{k}, k \in K$ contains the input quantities that are directly allocated to the production of the output $k$, while the remaining component $\mathbf{x}_{s+1}$ captures the jointly used input. Evidently, each $\hat{\mathbf{x}}=\left(\begin{array}{llll}\mathbf{x}_{1}^{\prime} & \ldots & \mathbf{x}_{s}^{\prime} & \mathbf{x}_{s+1}^{\prime}\end{array}\right)^{\prime}$ defines a unique $\mathbf{x}=\sum_{l=1}^{s+1} \mathbf{x}_{l}$; we will repeatedly use this in our following discussion.

Example 1. To illustrate the concept, we consider a situation where three inputs $(m=3)$ are used for the production of two outputs $(s=2)$. Suppose a firm with input vector $\mathbf{x}=\left(\begin{array}{lll}6 & 5 & 2\end{array}\right)$. Given that we have two outputs, we can define the corresponding decomposed input vector as $\hat{\mathbf{x}}=\left(\begin{array}{llll}\mathbf{x}_{1}^{\prime} & \mathbf{x}_{2}^{\prime} & \mathbf{x}_{3}^{\prime}\end{array}\right)^{\prime}$; the components $\mathbf{x}_{1}$ and $\mathbf{x}_{2}$ then capture the input used for the respective outputs 1 and 2 , and $\mathbf{x}_{3}$ contains the jointly used input. A feasible specification is $\mathbf{x}_{1}=\left(\begin{array}{lll}2 & 1 & 1\end{array}\right)^{\prime}, \mathbf{x}_{2}=\left(\begin{array}{lll}3 & 2 & 1\end{array}\right)^{\prime}$ and $\mathbf{x}_{3}=\left(\begin{array}{lll}1 & 2 & 0\end{array}\right)^{\prime}$. [Note that this specification effectively satisfies $\mathbf{x}=\sum_{l=1}^{3} \mathbf{x}_{l}$ but, of course, many other specifications are equally feasible.] In words, this specification implies that respectively 2 and 3 units of the input 1 are used for the production of the outputs 1 and 2, while 1 unit of the input 1 is jointly used; a directly similar interpretation holds for the inputs 2 and 3.

At this point, we stress that we usually cannot observe the exact allocation of the observed (aggregate) input vector $\mathbf{x}$ to its constituent components $\mathbf{x}_{1}, \ldots, \mathbf{x}_{s+1}$; the mere restriction is that, for each observation $i$, the components $\mathbf{x}_{l}^{i}$ must sum up to the observed input vector $\mathbf{x}^{i}$. Note further that it may well be that some components of the decomposed input vector $\hat{\mathbf{x}}$ equal zero; e.g., there may be no joint input use, which means that $\mathbf{x}_{s+1}$ is a zero vector. [Such 
cases, which include additional information, may entail more stringent cost efficiency conditions. We return to this in the concluding discussion.]

We describe the technology in terms of input requirement sets $V_{k}\left(y_{k}\right) \subseteq\left(\Re_{+}^{m}\right)^{s+1}$ associated with the $k$-th output quantity $y_{k}$ (i.e. the $k$-th entry of the output vector $\mathbf{y}$ ); the statement $\hat{\mathbf{x}} \in V_{k}\left(y_{k}\right)$ then indicates that the decomposed input vector $\hat{\mathbf{x}}$ produces at least the output quantity $y_{k}$. This specification of the sets $V_{k}\left(y_{k}\right)$ effectively implies that the production of each output $k$ may depend not only on the input $\mathbf{x}_{k}$ specific to the production of the $k$-th output but also, through production externalities, on the inputs $\mathbf{x}_{k^{*}}$ that are allocated to some other output $k^{*}$, and on the jointly used input $\mathbf{x}_{s+1}$. By construction, the input requirement sets are nested in the following sense: ${ }^{3}$

$$
\left(\hat{\mathbf{x}} \in V_{k}\left(y_{k}\right) \wedge y_{k} \geq \tilde{y}_{k}\right) \Rightarrow \hat{\mathbf{x}} \in V_{k}\left(\tilde{y}_{k}\right)
$$

this is a standard condition which reflects that less output never requires more input or, in other words, that outputs are freely disposable.

The presence of production externalities and jointly used inputs makes it impossible to consider each output separately in the cost efficiency analysis; e.g., the specification of the (unobserved) decomposed input vector $\hat{\mathbf{x}} \in V_{k}\left(y_{k}\right)$ most clearly reveals that the input requirement sets associated with different outputs are mutually interdependent. To obtain a setting that allows for analyzing cost efficiency at the (multi-output) firm level, we define the set $\mathbf{V}(\mathbf{y})$, which contains all decomposed input vectors $\hat{\mathbf{x}}$ that can produce the multi-valued output vector $\mathbf{y}$. The formal interrelationship between the input requirement set $\mathbf{V}(\mathbf{y})$ and the sets $V_{k}\left(y_{k}\right)$ is as follows:

$$
\text { for } \mathbf{y}=\left(\begin{array}{lll}
y_{1} & \ldots & y_{s}
\end{array}\right)^{\prime}: \hat{\mathbf{x}} \in \mathbf{V}(\mathbf{y}) \Leftrightarrow \forall k \in K: \hat{\mathbf{x}} \in V_{k}\left(y_{k}\right) \text {. }
$$

Using $\mathbf{V}(\mathbf{y})$, we can next define the further production assumption of convexity in output space. We define this property in terms of some given budget $z$ that can be used for purchasing the inputs (under the prices $\mathbf{p}$ ):

$$
\begin{aligned}
& \left(\exists \hat{\mathbf{x}}^{a}: \mathbf{p} \mathbf{x}^{a} \leq z \wedge \hat{\mathbf{x}}^{a} \in \mathbf{V}\left(\mathbf{y}^{a}\right)\right) \wedge\left(\exists \hat{\mathbf{x}}^{b}: \mathbf{p} \mathbf{x}^{b} \leq z \wedge \hat{\mathbf{x}}^{b} \in \mathbf{V}\left(\mathbf{y}^{b}\right)\right) \\
& \Rightarrow \forall \lambda \in[0,1]:\left(\exists \hat{\mathbf{x}}^{c}: \mathbf{p} \mathbf{x}^{c} \leq z \wedge \hat{\mathbf{x}}^{c} \in \mathbf{V}\left(\lambda \mathbf{y}^{a}+(1-\lambda) \mathbf{y}^{b}\right)\right) .
\end{aligned}
$$

In words, this definition states that, if the budget $z$ can afford the production of $\mathbf{y}^{a}$ and $\mathbf{y}^{b}$ (through the decomposed input vectors $\hat{\mathbf{x}}^{a}$ and $\hat{\mathbf{x}}^{b}$, respectively) then it can also produce any convex combination of these output vectors (through some decomposed input vector $\hat{\mathbf{x}}^{c}$ ). The economic interpretation of the condition is that the marginal rates of output transformation are

\footnotetext{
${ }^{3}$ As discussed by Varian (1984), including this condition avoids trivial rationalizations of the data in the sense of the following Definition 1 .
} 
everywhere decreasing (or, stricto sensu, non-increasing) along the boundary of the output producible set associated with the budget $z .^{4}$

Using this convexity property, we can characterize efficient production behavior. As a preliminary step, we note that each input requirement set $V_{k}\left(y_{k}\right)$ corresponds to a production function $f_{k}(\hat{\mathbf{x}})$, which gives the maximum quantity of the output $k$ that can be produced with $\hat{\mathbf{x}}$. We have:

$$
\hat{\mathbf{x}} \in V_{k}\left(y_{k}\right) \Leftrightarrow f_{k}(\hat{\mathbf{x}}) \geq y_{k}
$$

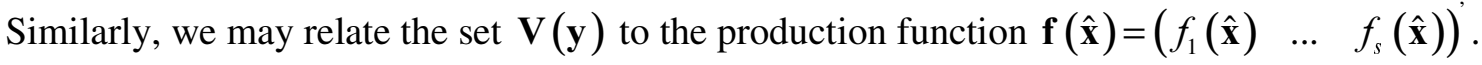

In our approach, cost efficient production behavior means that, for each firm observation $i$, the selected decomposed input vector $\hat{\mathbf{x}}^{i}$ yields an output combination $\mathbf{y}^{i}$ that is situated on the efficient boundary of the (convex) set of producible output combinations associated with the given budget (which corresponds to the observed outlay $\left(\left(\mathbf{p}^{i}\right)^{\prime} \mathbf{x}^{i}\right)$ ); this effectively represents a 'rational' allocation of the available budget. To formally define the condition, we use a standard result in welfare economics, namely: under convex utility possibility sets, any Pareto-efficient allocation can be characterized as a stationary point of a linear social welfare function (see e.g. Mas-Colell et al., 1995). This result is readily translated towards the current setting, which is characterized by convex output producible sets (instead of utility possibility sets). More specifically, we obtain that efficient production behavior requires that each observation $i$ maximizes a multi-output production function (linear in terms of $\mathbf{y}^{i}$ ) for the observed outlay, i.e.:

$$
\forall i \in S, \exists \boldsymbol{\mu}^{i} \in \mathfrak{R}_{+}^{s}:\left(\boldsymbol{\mu}^{i}\right)^{\prime} \mathbf{y}^{i}=\max _{\hat{\mathbf{x}}}\left\{\left(\boldsymbol{\mu}^{i}\right)^{\prime} \mathbf{f}(\hat{\mathbf{x}}) \mid \mathbf{x} \in \Re_{+}^{m} \text { with }\left(\mathbf{p}^{i}\right)^{\prime} \mathbf{x} \leq\left(\mathbf{p}^{i}\right)^{\prime} \mathbf{x}^{i}\right\}
$$

In this cost efficiency condition, the vector $\boldsymbol{\mu}^{i}$ can be interpreted as priority weight vector, with the different entries representing the weights that the firm under evaluation attributes to the different outputs. These weights correspond to some implicit (possibly nonlinear) production objective function that aggregates the different individual outputs, and which underlies the observed output choices. Importantly, these priority weights need not be constant across the firm observations: they can change depending on the economic circumstances. For example, if the valuation of some output $k$ increases, then the firm may want to increase (in relative terms) the production of the output $k$, which is translated in a higher priority weight. The analogy with the Pareto efficiency concept in welfare economics is immediate: cost-efficient behavior, for a given budget/outlay, implies that it is impossible to increase any output $k$ without decreasing another output $k^{*}$.

The question of data consistency with the cost efficiency condition is then whether it is possible to conceive a collection of input requirement sets that makes observed behavior consistent with the above efficiency condition. The following definition states the formal conditions for such a cost rationalization (or $C-R$ ) of the production data:

\footnotetext{
${ }^{4}$ It can be verified that this output convexity condition is actually somewhat stronger than that forwarded by Petersen (1990) and Bogetoft (1996) in a similar context. The more stringent property is essential for obtaining the cost efficiency condition in (2.1), which will form the basis for our nonparametric characterization of multioutput production under economies of scope.
} 
Definition 1. For a production process characterized by production externalities and joint input use, a family of input requirement sets $\left\{V_{k}\left(y_{k}\right), k \in K\right\}$ provides a $\mathrm{C}-\mathrm{R}$ of the set $\left\{\left(\mathbf{p}^{i}, \mathbf{x}^{i}, \mathbf{y}^{i}\right) \mid i \in S\right\}$ if there exists a production function $\mathbf{f}(\hat{\mathbf{x}})$, such that for each $i \in S$ there exist a decomposed input vector $\hat{\mathbf{x}}^{i}=\left(\begin{array}{llll}\left(\mathbf{x}_{1}^{i}\right)^{\prime} & \ldots & \left(\mathbf{x}_{s}^{i}\right)^{\prime} & \left.\left(\mathbf{x}_{s+1}^{i}\right)^{\prime}\right)^{\prime}\end{array}\right)^{\prime}$ and a priority weight vector $\boldsymbol{\mu}^{i}$ that satisfy:

$$
\begin{aligned}
& \text { 1. } \mathbf{f}\left(\hat{\mathbf{x}}^{i}\right)=\mathbf{y}^{i} \\
& \text { 2. }\left(\boldsymbol{\mu}^{i}\right)^{\prime} \mathbf{f}\left(\hat{\mathbf{x}}^{i}\right) \geq\left(\boldsymbol{\mu}^{i}\right)^{\prime} \mathbf{f}(\hat{\mathbf{x}}) \text { for all } \hat{\mathbf{x}}=\left(\begin{array}{llll}
\mathbf{x}_{1}^{\prime} & \ldots & \mathbf{x}_{s}^{\prime} & \mathbf{x}_{s+1}^{\prime}
\end{array}\right)^{\prime} \in\left(\Re_{+}^{m}\right)^{s+1} \text { with }\left(\mathbf{p}^{i}\right)^{\prime} \mathbf{x} \leq\left(\mathbf{p}^{i}\right)^{\prime} \mathbf{x}^{i} .
\end{aligned}
$$

In words, this definition requires that there must exist at least one feasible decomposition of the observed input vectors $\mathbf{x}^{i}$ into decomposed input vectors $\hat{\mathbf{x}}^{i}$ and, accordingly, priority weight vectors $\boldsymbol{\mu}^{i}$ and some production function $\mathbf{f}(\hat{\mathbf{x}})$ such that each firm observation $i$ is consistent with the efficiency condition (2.1). This efficiency condition cannot be used as such, since the production technology (and, hence, the function $\mathbf{f}(\hat{\mathbf{x}}))$ is typically unknown. Essentially, the nonparametric approach to analyzing production behavior focuses on efficiency conditions that do not necessitate a (non-verifiable) functional specification of the production possibilities.

To provide a nonparametric characterization of cost efficient behavior in the sense of Definition 1, we first define the additional concept of implicit price vectors $\left(\hat{\mathbf{p}}_{1}, \ldots, \hat{\mathbf{p}}_{s}\right)$ for an (aggregate) input price vector $\mathbf{p}$ as

$$
\begin{aligned}
& \forall k \in K: \quad \hat{\mathbf{p}}_{k}=\left(\begin{array}{llll}
\boldsymbol{\pi}_{k, 1}^{\prime} & \ldots & \boldsymbol{\pi}_{k, s}^{\prime} & \boldsymbol{\pi}_{k, s+1}^{\prime}
\end{array}\right)^{\prime} \text { such that } \\
& \forall l \in\{1, \ldots, s+1\}: \boldsymbol{\pi}_{k, l} \in \mathfrak{R}_{+}^{m} \quad \text { with } \quad \sum_{k \in K} \boldsymbol{\pi}_{k, l}=\mathbf{p} .
\end{aligned}
$$

This concept complements the earlier concept of decomposed input vector $\hat{\mathbf{x}}$ : each $\hat{\mathbf{p}}_{k}=\left(\begin{array}{llll}\boldsymbol{\pi}_{k, 1} & \ldots & \boldsymbol{\pi}_{k, s}^{\prime} & \boldsymbol{\pi}_{k, s+1}^{\prime}\end{array}\right)^{\prime}$ captures the fraction of the price for the decomposed input quantities $\hat{\mathbf{x}}=\left(\begin{array}{llll}\mathbf{x}_{1} & \ldots & \mathbf{x}_{s}^{\prime} & \mathbf{x}_{s+1}^{\prime}\end{array}\right)^{\prime}$ that is attributed to the output $k$. To see this, we first recall that the decomposition of the (aggregate) input vector $\mathbf{x}$ into $s+1$ components $\mathbf{x}_{l}$ effectively reveals the different channels through which the observed inputs are allocated. Correspondingly, each $l$-th component $\boldsymbol{\pi}_{k, l}$ of the implicit price vectors $\hat{\mathbf{p}}_{k}$ gives the fraction of the price of each input component $\mathbf{x}_{l}$ that is attributed to the output $k{ }^{5}$ More specifically, the components $\boldsymbol{\pi}_{k, k}$ capture the fraction of the price of the input directly allocated to output $k$ that is effectively borne by that output $k$. Next, the components $\pi_{k, k^{*}}\left(k^{*} \in K, k^{*} \neq k\right)$ refer to the possibility of input externalities: $\boldsymbol{\pi}_{k, k^{*}} \neq \mathbf{0}$ means that the inputs allocated to the output $k^{*}$ $\left(\mathbf{x}_{k^{*}}\right)$ benefit the production of the output $k$ (which is thus compensated through $\boldsymbol{\pi}_{k, k^{*}}$ ).

\footnotetext{
${ }^{5}$ In fact, the intuition of the implicit price vectors is analogous to that of Lindahl prices in the context of public goods. The concept of Lindahl prices is mainly used in a public economics context; see, e.g., Myles (1995) for a discussion.
} 
Finally, as for the jointly used input $\mathbf{x}_{s+1}$, the cost must be distributed over the different outputs (see in particular $\sum_{k \in K} \boldsymbol{\pi}_{k, s+1}=\mathbf{p}$ ).

Example 2. To further illustrate the concept, we recapture the situation with three inputs and two outputs in Example 1. Suppose the input price vector $\mathbf{p}=\left(\begin{array}{lll}2 & 1 & 3\end{array}\right)$. Given that we have two outputs, we can define implicit price vectors $\hat{\mathbf{p}}_{1}=\left(\begin{array}{llll}\boldsymbol{\pi}_{1,1}^{\prime} & \boldsymbol{\pi}_{1,2}^{\prime} & \boldsymbol{\pi}_{1,3}^{\prime}\end{array}\right)^{\prime}$ and $\hat{\mathbf{p}}_{2}=\left(\begin{array}{lll}\boldsymbol{\pi}_{2,1}^{\prime} & \boldsymbol{\pi}_{2,2}^{\prime} & \boldsymbol{\pi}_{2,3}^{\prime}\end{array}\right)^{\prime}$. A feasible specification is the following:

$$
\boldsymbol{\pi}_{1,1}=\left(\begin{array}{c}
1.5 \\
0.5 \\
1.5
\end{array}\right), \boldsymbol{\pi}_{1,2}=\left(\begin{array}{c}
0 \\
0.5 \\
2
\end{array}\right), \boldsymbol{\pi}_{1,3}=\left(\begin{array}{c}
1 \\
0 \\
1.5
\end{array}\right) ; \text { and } \boldsymbol{\pi}_{2,1}=\left(\begin{array}{c}
0.5 \\
0.5 \\
1.5
\end{array}\right), \boldsymbol{\pi}_{2,2}=\left(\begin{array}{c}
2 \\
0.5 \\
1
\end{array}\right), \boldsymbol{\pi}_{2,3}=\left(\begin{array}{c}
1 \\
1 \\
1.5
\end{array}\right)
$$

In words, $\boldsymbol{\pi}_{1,1} \neq \mathbf{p}$ implies that the output 1 does not fully bear the cost for the input used for its production $\left(\mathbf{x}_{1}\right)$; this reflects that input externalities benefit the production of the output 2 (for which the compensation is captured by $\boldsymbol{\pi}_{2,1}\left(=\left(\mathbf{p}-\boldsymbol{\pi}_{1,1}\right)\right.$ ). Similarly, the input used for the production of the output $2\left(\mathbf{x}_{2}\right)$ implies production externalities towards the production of the output 1 (see the specification of $\boldsymbol{\pi}_{1,2}$ and $\boldsymbol{\pi}_{2,2}$ ); in this case there are no externalities associated with the input 1 (as the first entry of $\boldsymbol{\pi}_{1,2}$ is zero). Finally, the remaining components $\boldsymbol{\pi}_{1,3}$ and $\boldsymbol{\pi}_{2,3}$ distribute the cost of the jointly used input.

As a final note, we indicate that a specification of the decomposed input vector $\hat{\mathbf{x}}$ and the implicit price vectors $\left(\hat{\mathbf{p}}_{1}, \ldots, \hat{\mathbf{p}}_{s}\right)$ effectively allows for computing the cost share attributed to each output $k$ (as $\hat{\mathbf{p}}_{k} \hat{\mathbf{x}}$ ). As for our example, the given specification of $\hat{\mathbf{x}}$ (in Example 1), $\hat{\mathbf{p}}_{1}$ and $\hat{\mathbf{p}}_{2}$ implies a cost level of $9\left(=\hat{\mathbf{p}}_{1}^{\prime} \hat{\mathbf{x}}=\boldsymbol{\pi}_{1,1}^{\prime} \mathbf{x}_{1}+\boldsymbol{\pi}_{1,2}^{\prime} \mathbf{x}_{2}+\boldsymbol{\pi}_{1,3}^{\prime} \mathbf{x}_{3}\right)$ for the output 1 and a cost level of $14\left(=\hat{\mathbf{p}}_{2}^{\prime} \hat{\mathbf{x}}=\boldsymbol{\pi}_{2,1}^{\prime} \mathbf{x}_{1}+\boldsymbol{\pi}_{2,2}^{\prime} \mathbf{x}_{2}+\boldsymbol{\pi}_{2,3}^{\prime} \mathbf{x}_{3}\right)$ for the output 2 . Note that the sum of these cost shares equals the total cost of production $\mathbf{p} \mathbf{x}$.

Once more we should stress that, for a given observation $i$, we usually cannot observe the

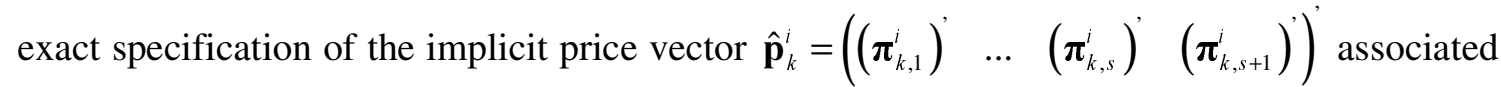
with each output $k$. The only restriction is that the components $\boldsymbol{\pi}_{k, l}^{i}$ must add up (over the different outputs $k$ ) to the observed (aggregate) price, i.e. $\sum_{k \in K} \boldsymbol{\pi}_{k, l}^{i}=\mathbf{p}^{i}$. [We return to the use of additional information regarding the specification of the implicit price vectors $\left(\hat{\mathbf{p}}_{1}, \ldots, \hat{\mathbf{p}}_{s}\right)$ in the concluding discussion.]

We are now in a position to state a nonparametric necessary and sufficient condition for cost efficient production behavior in the sense of Definition $1:^{6}$

\footnotetext{
${ }^{6}$ In Proposition 1, positive monotonicity of a set $V_{k}\left(y_{k}\right)$ means that $\left(\hat{\mathbf{x}} \in V_{k}\left(y_{k}\right) \wedge \hat{\mathbf{x}}^{*} \geq \hat{\mathbf{x}}\right) \Rightarrow \hat{\mathbf{x}}^{*} \in V_{k}\left(y_{k}\right)$. This is essentially the property of free input disposal, i.e. more input can always produce the same output.
} 
Proposition 1. For a production process characterized by production externalities and joint input use, there is a family of closed, convex, positive monotonous input requirement sets that provide $a \mathrm{C}-\mathrm{R}$ of the data if and only if for each $i \in S$ there exists

1. $a$ decomposed input vector $\hat{\mathbf{x}}^{i}=\left(\begin{array}{llll}\left(\mathbf{x}_{1}^{i}\right)^{\prime} & \ldots & \left(\mathbf{x}_{s}^{i}\right)^{\prime} & \left.\left(\mathbf{x}_{s+1}^{i}\right)^{\prime}\right)^{\prime}\end{array}\right)^{\prime}$ and

2. implicit price vectors $\left(\hat{\mathbf{p}}_{1}^{i}, \ldots, \hat{\mathbf{p}}_{s}^{i}\right)$

such that for each $i, j \in S, k \in K:\left(y_{k}^{j} \geq y_{k}^{i}:\left(\hat{\mathbf{p}}_{k}^{i}\right)^{\prime} \hat{\mathbf{x}}^{j} \geq\left(\hat{\mathbf{p}}_{k}^{i}\right)^{\prime} \hat{\mathbf{x}}^{i}\right)$.

Hence, consistency with the cost efficiency condition requires that observed behavior satisfies a number of cost minimization conditions (i.e., one for each individual output $k$ ) that are expressed in terms of decomposed input vectors $\hat{\mathbf{x}}^{i}$ and corresponding implicit price vectors $\left(\hat{\mathbf{p}}_{1}^{i}, \ldots, \hat{\mathbf{p}}^{i}\right)$ for each observation $i$ : if observation $j$ produces more of the output $k$ than observation $i\left(y_{k}^{j} \geq y_{k}^{i}\right)$, then cost efficiency requires that the $k$-th output cost (under the prices $\hat{\mathbf{p}}_{k}^{i}$ ) for observation $i$ does not exceed that for observation $j$ (or, $\left.\left(\hat{\mathbf{p}}_{k}^{i}\right)^{\prime} \hat{\mathbf{x}}^{j} \geq\left(\hat{\mathbf{p}}_{k}^{i}\right)^{\prime} \hat{\mathbf{x}}^{i}\right)$. $^{7}$ Our above explanation of the vectors $\hat{\mathbf{x}}^{i}$ and $\left(\hat{\mathbf{p}}_{1}^{i}, \ldots, \hat{\mathbf{p}}^{i}\right)$ makes clear that this nonparametric characterization has a natural intuition in terms of the underlying model of joint input use and production externalities.

\section{Empirical tests of cost efficient behavior under economies of scope}

The $C-R$ condition in Proposition 1 is expressed in terms of decomposed input vectors $\hat{\mathbf{x}}^{i}$ and implicit price vectors $\left(\hat{\mathbf{p}}_{1}^{i}, \ldots, \hat{\mathbf{p}}^{i}\right)$. Empirical testing of the condition would be easy if these vectors were observed. However, this quantity and price information is usually not available, which makes direct empirical implementation of the $C-R$ conditions generally hard in computational terms. Specifically, one cannot conclude whether a $C-R$ of a given data set is possible by using an algorithm that tries to find decomposed input vectors and implicit price vectors that are consistent with the conditions in Proposition 1: in general, no algorithm can exhaust all feasible values and combinations of these unobservables. Therefore, in this section we formulate necessity and sufficiency conditions that solely include the (aggregate) observed input quantity and price information.

We first present the necessity condition. Before formulating that condition, we define an output dominating reference set $R^{i}$ for observation $i$ as

$$
R^{i}=\left\{j^{k} \in S \mid \forall k \in K, \exists j^{k}: y_{k}^{j^{k}} \geq y_{k}^{i}\right\}
$$

In words, each set $R^{i}$ is constructed such that, for each output $k$, it contains at least one observation $j^{k} \in R^{i}$ that dominates observation $i$ in that output $k\left(y_{k}^{j^{k}} \geq y_{k}^{i}\right)$. For each observation $i$,

\footnotetext{
${ }^{7}$ Interestingly, these cost minimization conditions are formally analogous to the condition derived by Varian (1984; Theorem 1) in the single output case. Given our multi-output orientation, we identify a separate condition for each entry $k \in K$ of the evaluated output vector $\mathbf{y}^{i}$. In fact, if there is only a single output $(s=1)$ then the nonparametric cost rationalization condition in Proposition 1 effectively reduces to Varian's condition.
} 
$\mathbf{R}^{i}$ is the collection of output dominating reference sets $R^{i}$.

Example 3. We illustrate by means of three observations ( $S=\{1,2,3\}$ ) that are taken from our own empirical application (see Section 4), which considers a situation with three outputs $(s=3)$. We have:

$$
\mathbf{y}^{1}=\left(\begin{array}{l}
1 \\
27 \\
4
\end{array}\right), \mathbf{y}^{2}=\left(\begin{array}{l}
1 \\
27 \\
2
\end{array}\right) \text { and } \mathbf{y}^{3}=\left(\begin{array}{l}
0 \\
13 \\
5
\end{array}\right)
$$

Let us construct the output dominating reference sets for observation 1. Trivially, one such set is the singleton $\{1\}$. Evidently, this implies that the sets $\{1,2\},\{1,3\}$ and $\{1,2,3\}$ equally satisfy the definition of output dominating reference set for observation 1. [More generally, we have that, if $R_{1}^{i} \subseteq S$ is an output dominating reference set for observation $i$, then by construction any larger subset $R_{2}^{i} \subseteq S$ (with $R_{1}^{i} \subseteq R_{2}^{i}$ ) is an output dominating reference set for the same observation.] In addition, given the output vectors of observations 2 and 3 a final output dominating reference set is the pair $\{2,3\}$ (because $y_{1}^{2} \geq y_{1}^{1}, \quad y_{2}^{2} \geq y_{2}^{1}$ and $\left.y_{3}^{3} \geq y_{3}^{1}\right)$. Hence, we obtain the collection of output dominating reference sets

$$
\mathbf{R}^{1}=\{\{1\},\{1,2\},\{1,3\},\{1,2,3\},\{2,3\}\}
$$

We can now define the nonparametric necessary condition for cost efficient production that solely includes aggregate price and quantity information:

Proposition 2. For a production process characterized by production externalities and joint input use, there is a family of closed, convex, positive monotonous input requirement sets that provide a C-R of the data only if for each $i \in S:\left(\mathbf{p}^{i}\right)^{\prime} \mathbf{x}^{i} \leq \min _{R^{i} \in \mathbf{R}^{i}}\left(\mathbf{p}^{i}\right)^{\prime}\left(\sum_{j \in R^{i}} \mathbf{x}^{j}\right)$.

This condition compares the cost level $\left(\mathbf{p}^{i}\right)^{\prime} \mathbf{x}^{i}$ for the evaluated firm observation $i$ to the cost level for each combination of observations $j^{k} \in S$ with $y_{k}^{j^{k}} \geq y_{k}^{i}$ for each output $k \in K$; see the construction of the output dominating reference sets $R^{i}$. More specifically, the condition states that the cost level of $i$ should not exceed, under the prices that apply to $i$, the cost level for the (sum) input vector $\sum_{j \in R^{i}} \mathbf{x}^{j}$ associated with any set $R^{i} \in \mathbf{R}^{i}$. Intuitively, if this condition were not met, then observation $i$ could have produced (at least) the same output vector $\mathbf{y}^{i}$ at a lower cost level by using the (sum) input vector $\sum_{j \in R^{i}} \mathbf{x}^{j}$ instead of the chosen vector $\mathbf{x}^{i}$.

If an observation $i$ does not meet the corresponding necessary condition for cost efficient behavior, then it is useful to quantify the corresponding deviation from the (necessary) efficiency condition. To do so, we use a cost efficiency measure defined as the ratio of the minimum cost level needed to obtain consistency with the necessity requirement over the actual cost level: 


$$
\varphi^{i}=\frac{\min _{R^{i} \in \mathbf{R}^{i}}\left(\mathbf{p}^{i}\right)^{\prime}\left(\sum_{j \in R^{i}} \mathbf{x}^{j}\right)}{\left(\mathbf{p}^{i}\right)^{\prime} \mathbf{x}^{i}}
$$

The corresponding necessary condition for a $C-R$ of the data to be possible is that $\varphi^{i}=1$ for each $i \in S$. The value $\varphi^{i}<1$ captures the extent to which costs should (minimally) be reduced in order to obtain consistency of the observation $i$ with the cost rationalization conditions. From that perspective, the measure $\varphi^{i}$ reveals for each individual firm observation the degree of consistency with the necessary $C-R$ requirement in Proposition 2 , and may thus be interpreted as a goodness-of-fit measure for the cost efficiency condition under investigation. See Varian (1990) and Färe and Grosskopf (1995) for a detailed discussion of this goodnessof-fit idea in a similar context of nonparametric production analysis.

Example 4. To further illustrate, we recapture the situation in Example 3. Specifically, we evaluate observation 1 and use the additional information (again taken from our empirical application; see Section 4) that the three observations produce the three outputs by means of two inputs $(m=2)$ with quantities and prices

$$
\mathbf{x}^{1}=\left(\begin{array}{l}
28.2 \\
23
\end{array}\right), \mathbf{x}^{2}=\left(\begin{array}{l}
7.2 \\
9.2
\end{array}\right), \mathbf{x}^{3}=\left(\begin{array}{l}
10.2 \\
8
\end{array}\right) ; \text { and } \mathbf{p}^{1}=\left(\begin{array}{l}
1798.660 \\
3129.605
\end{array}\right)
$$

On the one hand, we find that $\left(\mathbf{p}^{1}\right)^{\prime} \mathbf{x}^{1}=122933.115$. On the other hand, it can be verified that $\min _{R^{1} \in \mathbf{R}^{1}}\left(\mathbf{p}^{1}\right)^{\prime}\left(\sum_{j \in R^{1}} \mathbf{x}^{j}\right)=\left(\mathbf{p}^{1}\right)^{\prime}\left(\mathbf{x}^{2}+\mathbf{x}^{3}\right)=85289$. Hence, $\left(\mathbf{p}^{1}\right)^{\prime} \mathbf{x}^{1}>\min _{R^{1} \in \mathbf{R}^{1}}\left(\mathbf{p}^{1}\right)^{\prime}\left(\sum_{j \in R^{1}} \mathbf{x}^{j}\right)$ and, thus, observation 1 does not meet the necessary cost minimization condition in Proposition 1. The corresponding cost efficiency measure $\varphi^{1}=0.694$ suggests that, for the given output, observation 1 can reduce its cost level by (at least) $30.6 \%$. Obviously, $\varphi^{1}<1$ a fortiori implies that a $C-R$ of this data set is impossible.

Proposition 2 institutes a condition on the observable price and quantity information that should always be met by production processes consistent with the $C-R$ Definition 1 . Still, meeting this condition does not mean that there effectively exists a $C-R$ of the production data under consideration; i.e. the condition is necessary but not sufficient for a $C-R$ to be possible. A complementary sufficiency condition is:

Proposition 3. For a production process characterized by production externalities and joint input use, there is a family of closed, convex, positive monotonous input requirement sets that provide a $\mathrm{C}-\mathrm{R}$ of the data if it is possible to construct a partitioning $S_{k}(k \in K)$ with $\bigcup_{k \in K} S_{k}=S \quad$ and $\quad S_{k} \cap S_{k^{*}}=\varnothing \quad\left(k, k^{*} \in K, k \neq k^{*}\right) \quad$ such that for $i \in S_{k}$ :
$\left[\forall j \in S_{k}: y_{k}^{j} \geq y_{k}^{i} \Rightarrow\left(\mathbf{p}^{i}\right)^{\prime} \mathbf{x}^{i} \leq\left(\mathbf{p}^{i}\right)^{\prime} \mathbf{x}^{j}\right]$ and $\left[\forall j^{*} \in S_{k^{*}}, k^{*} \neq k: y_{k^{*}}^{j^{*}}>y_{k^{*}}^{i}\right]$

Intuitively, this condition considers the extreme scenario where each firm observation $i \in S_{k}$ allocates the total input exclusively to the production of a single output $k$. The first part of the closing cost minimization condition then states that it should not be possible to produce (at least) the associated output quantity $y_{k}^{i}$ at a lower cost when compared to any (similarly 
specialized) production plan $j$ with $y_{k}^{j} \geq y_{k}^{i}$. The second part of the closing condition imposes that, under such exclusive allocation (or specialization), the observation $i$ should not dominate some other observation $j^{*}$ in output $k^{*}$ if the latter observation effectively specializes in producing $k^{*}$. $^{8}$

Clearly, testing data consistency with the empirical requirements in Propositions 2 and 3 is a finite process because, essentially, for each observation $i$ the cardinality of the set of observations $j$ that dominate observation $i$ in at least one output $k$ (i.e. the set $\left.\left\{j \in S \mid \exists k \in K: y_{k}^{j} \geq y_{k}^{i}\right\}\right)$ is finite in nature. Given this, the necessity and sufficiency tests may be implemented by means of simple enumeration algorithms, which consecutively consider all feasible specifications of the sets $R^{i}$ for each observation $i$ (for the necessity requirement) and the sets $S_{k}$ for each output $k$ (for the sufficiency requirement). The next section provides an illustrative application with $s=3$.

Our empirical necessity and sufficiency requirements will in general not coincide; this discrepancy essentially reflects the unobservability of the decomposed input vectors and implicit price vectors in Proposition 1. The only instance in which both conditions are equivalent occurs when there is a single output $(s=1)$. The intuition is straightforward: in that case, the decomposed input vectors and implicit price vectors are the observed input and price vectors, and thus the necessity and sufficiency conditions for a $C-R$ of the data always coincide; or, from a different perspective, the empirical implications of joint input use and production externalities become irrelevant if there is only a single output.

In the general case (for $s \geq 2$ ), violation of the necessary condition in Proposition 2 means that a $C-R$ of the data is impossible, while consistency with the sufficient condition in Proposition 3 entails the opposite conclusion. As for data that meet the necessity but not the sufficiency condition, we cannot directly tell from the observable price and quantity information whether a $C-R$ of the data is effectively possible. In such cases, one may, for example, impose some additional prior structure on the decomposed input vectors and implicit price vectors.

Still, even though the necessary condition should not generally coincide with the sufficient condition, we may expect the two conditions to become equally powerful (or 'converge') when the sample size increases. Specifically, for large $t$ the probability increases that for $i \in S$ there exists $k \in K$ such that for all $k^{*} \in K, k^{*} \neq k$ we have that $\min _{j \in S}\left\{\left(\mathbf{p}^{i}\right)^{\prime} \mathbf{x}^{j} \mid y_{k^{*}}^{j} \geq y_{k^{*}}^{i}\right\}$ gets close to zero. In such a situation, the difference between $\min _{R^{i} \in \mathbf{R}^{i}} \sum_{j \in R^{i}}\left(\mathbf{p}^{i}\right)^{\prime} \mathbf{x}^{j}$ and $\min _{j \in S: y_{k}^{j} \geq y_{k}^{i}}\left(\mathbf{p}^{i}\right)^{\prime} \mathbf{x}^{j} \quad$ diminishes. As a result, the empirical requirement $\left(\mathbf{p}^{i}\right)^{\prime} \mathbf{x}^{i} \leq \min _{R^{i} \in \mathbf{R}^{i}} \sum_{j \in R^{i}}\left(\mathbf{p}^{i}\right)^{\prime} \mathbf{x}^{j}$ in Proposition 2 will approach the condition $\left(\mathbf{p}^{i}\right)^{\prime} \mathbf{x}^{i} \leq\left(\mathbf{p}^{i}\right)^{\prime} \mathbf{x}^{j}$ for $j \in S: y_{k}^{j} \geq y_{k}^{i}$ in Proposition $3 .^{9}$

\footnotetext{
${ }^{8}$ To avoid a conflict with the usual "no free lunch" assumption, one may also interpret the sufficiency condition in terms of quasi-exclusive input allocation (i.e. the production of a single output consumes almost all input while a minimal amount of input is allocated to each other output). [The proof in the appendix is easily accommodated.] More generally, it is worth stressing that, for data that are consistent with the sufficiency condition, this may not be the only data rationalizing interpretation. The sole implication of the sufficiency result is that (quasi-)exclusive input allocation always constitutes a possible interpretation.

${ }^{9}$ As for this last formulation of the sufficiency condition in Proposition 3, we note that the requirement $\left(\mathbf{p}^{i}\right)^{\prime} \mathbf{x}^{i} \leq\left(\mathbf{p}^{i}\right)^{\prime} \mathbf{x}^{j} \quad$ for $\quad j \in S_{k}: y_{k}^{j} \geq y_{k}^{i} \quad$ is equivalent to $\left(\mathbf{p}^{i}\right)^{\prime} \mathbf{x}^{i} \leq\left(\mathbf{p}^{i}\right)^{\prime} \mathbf{x}^{j} \quad$ for $\quad j \in S: y_{k}^{j} \geq y_{k}^{i}$ when
} 
The associated 'convergence rate' will then of course depend (positively) upon the input price-quantity variation in the data and, hence, we may expect it to increase with the number of inputs. For a given number of inputs, the speed of convergence will vary with the specific data generating process that underlies the aggregate production data, which in turn depends on the specific characteristics of the production process (see the production function $\mathbf{f}(\hat{\mathbf{x}})$ and the priority weight vector $\boldsymbol{\mu}^{i}$ in (2.1)). But, in general, we can safely argue that, for larger samples, the empirical implications of the fairly rudimentary allocation process underlying the sufficient condition will get closer to those of any more refined allocation process captured by the necessary condition.

So far, we have assumed that prices $\mathbf{p}^{i}$ for each firm observation $i$ are known. In many cases, such reliable price information is not available. ${ }^{10}$ Starting from Proposition 2, we may then formulate a necessary condition for a $C-R$ of the data, as follows:

Corollary 1. For a production process characterized by production externalities and joint input use, there is a family of closed, convex, positive monotonous input requirement sets that provide a C-R of the data only if there exists a collection of price vectors $\left\{\mathbf{p}^{i} \in \mathfrak{R}_{+}^{m} \backslash \mathbf{0} \mid i \in S\right\}$ such that for each $i \in S:\left(\mathbf{p}^{i}\right)^{\prime} \mathbf{x}^{i} \leq \min _{R^{i} \in \mathbf{R}^{i}}\left(\mathbf{p}^{i}\right)^{\prime}\left(\sum_{j \in R^{\prime}} \mathbf{x}^{j}\right)$.

The interpretation of the condition is as follows: in the absence of fully reliable price information, a necessary condition for data consistency with the $C-R$ conditions is that there exists, for each observation $i$, at least one (non-zero) input price vector that implies consistency with the condition in Proposition 2. From the perspective of the evaluated production plan, such a price vector may be conceived as 'most favorable' in that it effectively minimizes the cost inefficiency. In a certain sense, such a most favorable price vector may be interpreted as a shadow price vector that supports cost efficient behavior of the evaluated production vector.

Checking consistency with the necessary condition in Corollary 1 boils down to solving the following linear programming problem (for each observation $i$ ):

Primal

$$
\begin{aligned}
& \theta^{i}=\max u \\
& \text { s.t. } \\
& \left(\mathbf{p}^{i}\right)^{\prime} \mathbf{x}^{i}=1 \\
& u \leq\left(\mathbf{p}^{i}\right)^{\prime}\left(\sum_{j \in R^{i}} \mathbf{x}^{j}\right) \quad \forall R^{i} \quad \sum_{R^{i}} \lambda^{R^{i}}=1 \\
& u \in \mathfrak{R}, \quad \mathbf{p}^{i} \in \mathfrak{R}_{+}^{m}
\end{aligned}
$$

\section{Dual}

\footnotetext{
$\min _{j \in S}\left\{\left(\mathbf{p}^{i}\right)^{\prime} \mathbf{x}^{j} \mid y_{k^{*}}^{j} \geq y_{k^{*}}^{i}\right\}=0 \quad$ for $\quad$ all $\quad k^{*} \in K, \quad k^{*} \neq k$. This follows from $\forall i \in S_{k}$ : $\left(j^{*} \in S_{k^{*}}, k^{*} \neq k \Rightarrow y_{k^{*}}^{j^{*}}>y_{k^{*}}^{i}\right)$; see also our proof of Proposition 3 .

${ }^{10}$ See, e.g., Kuosmanen et al. (2006) for a discussion of instances where reliable price information is not readily available. Our application in Section 4 contains a further example.
} 
In the primal formulation of the problem, the price normalization $\left(\mathbf{p}^{i}\right)^{i} \mathbf{x}^{i}=1$ effectively implements the condition $\mathbf{p}^{i} \in \mathfrak{R}_{+}^{m} \backslash \mathbf{0}$ in Corollary 1. Recalling our above interpretation of the corollary, the model checks whether, subject to the price normalization, there exists a set of shadow prices that make the firm observation $i$ consistent with the empirical cost minimization condition. Just like the measure $\varphi^{i}$ that we defined before, the measure $\theta^{i}$ captures the degree of (shadow) cost efficiency of the observation $i$, and it can be interpreted as goodness-of-fit measure. Clearly, we have $\theta^{i} \geq \varphi^{i}$ and a necessary condition for cost efficient production behavior is that $\theta^{i}=1$ for each observation $i$.

The dual problem in (3.1) computes (radial) Farrell (1957) efficiency with respect to a monotone production technology with convexified input sets; convexification is taken over the sets $R^{i}$. In fact, this primal-dual formulation shows the close connection with the nonparametric efficiency measurement literature known as Data Envelopment Analysis (DEA; after Charnes et al., 1978). Specifically, the dual problem in (3.1) is formally similar to Bogetoft's (1996) DEA model that computes Farrell efficiency with respect to a similar production technology; the main difference is that we convexify over the summed input vectors $\sum_{j \in R^{i}} \mathbf{x}^{j}$, which results from our specific scope economies perspective. ${ }^{11}$ Our above discussion establishes the model as a tool for testing data consistency with (shadow) cost efficiency.

Example 5. To illustrate, we recapture Example 4, but now we do not use the input price information. Again, we evaluate observation 1. For the given collection of output dominating reference sets $\mathbf{R}^{1}$ (reported in Example 3), the problems in (3.1) take the form

Primal

$$
\begin{aligned}
& \theta^{1}=\max u \\
& \text { s.t. } \\
& \left(\mathbf{p}^{1}\right)^{\prime} \mathbf{x}^{1}=1 \\
& u \leq\left(\mathbf{p}^{1}\right)^{\prime}\left(\mathbf{x}^{1}\right) \\
& u \leq\left(\mathbf{p}^{1}\right)^{\prime}\left(\mathbf{x}^{1}+\mathbf{x}^{2}\right) \\
& u \leq\left(\mathbf{p}^{1}\right)^{\prime}\left(\mathbf{x}^{1}+\mathbf{x}^{3}\right) \\
& u \leq\left(\mathbf{p}^{1}\right)^{\prime}\left(\mathbf{x}^{1}+\mathbf{x}^{2}+\mathbf{x}^{3}\right) \\
& u \leq\left(\mathbf{p}^{1}\right)^{\prime}\left(\mathbf{x}^{2}+\mathbf{x}^{3}\right) \\
& u \in \mathfrak{R}, \quad \mathbf{p}^{1} \in \mathfrak{R}_{+}^{m}
\end{aligned}
$$

\section{Dual}

The outcome is $\theta^{1}=0.748$. This means that, even when using the most favorable prices (in casu the computed shadow price vector $\left(\mathbf{p}^{1}\right)^{\prime}=\left(\begin{array}{lll}0.000 & 0.043\end{array}\right)$, we can identify a

\footnotetext{
11 Hanoch and Rothschild (1972; Section 2) introduced a similar production technology representation as Bogetoft (1996) in a setting involving multiple inputs and a single output.
} 
potential cost reduction of (at least) $25.2 \%$ for the observation $1 .^{12}$ The corresponding minimum cost level is associated with the (sum) input vector $\left(\mathbf{x}^{2}+\mathbf{x}^{3}\right)$ (or, for the dual problem we obtain $\lambda^{5}=1$ ).

To conclude this illustration, we note that the specification of the set $\mathbf{R}^{\mathrm{i}}$ may be fine-tuned to enhance the efficiency of the empirical testing of the condition. For example, we may exclude from consideration an output dominating reference set $R_{2}^{i} \in \mathbf{R}^{i}$ if there exist another set $R_{1}^{i} \in \mathbf{R}^{i}$ such that $R_{1}^{i} \subseteq R_{2}^{i}$ as, evidently, $\mathbf{p}\left(\sum_{j \in R_{2}^{i}} \mathbf{x}^{j}\right) \geq \mathbf{p}\left(\sum_{j \in R_{1}^{\prime}} \mathbf{x}^{j}\right)$ for any input price vector $\mathbf{p}$. For the specific data structure under investigation, this means that we can effectively restrict attention to $\{\{1\},\{2,3\}\} \subset \mathbf{R}^{1}$ when evaluating observation 1 . As a matter of fact, we have used this insight for the computations of our own application presented in the next section.

As a final note, we indicate that the computed shadow price vector $\mathbf{p}^{i}$ in (3.1) (and, correspondingly, the $\lambda^{R^{i}}$ in the dual problem) should in general not be unique: there may be multiple input price vectors that support the same cost efficiency level. Therefore, we choose not to focus on these shadow price estimates in our following empirical application.

\section{Illustrative application}

We apply the presented methodology for examining the behavior of research programs in Economics and Business Management faculties of Dutch universities. Specifically, we evaluate the efficiency of 77 research programs organized at 8 universities. The same data set was studied by Cherchye and Vanden Abeele (2005), who motivate efficiency assessment within this setting by the argument that efficient research production is not guaranteed by the usual market correction mechanisms. These authors further claim that a cost efficiency evaluation model is particularly appropriate within this application context. But they focus on a different cost efficiency criterion, which does not explicitly incorporate the empirical implications of joint input use and production externalities. Still, as we argued in the Introduction, the production process of university research seems well-suited to illustrate our method for assessing cost efficiency under scope economies. [In fact, this method implies a strengthened efficiency test as compared to that used by Cherchye and Vanden Abeele; we return to this below.]

Generally, a research program can be defined as "a group of researchers who join forces to investigate a particular theme, and in the process to educate researchers and to publish research results". Cherchye and Vanden Abeele argue that this definition institutes research programs as the natural production units for studying academic research efficiency. Building on that definition, they suggest the following input-output selection for characterizing the production of each program:

\footnotetext{
${ }^{12}$ The zero shadow price for the first input in this example is commonly referred to as a 'slack problem' in the DEA literature (e.g., Cooper et al., 2000). In this respect, we indicate that the problems in (3.1) may be enriched by adding additional restrictions on the relative price that incorporate a priori information regarding feasible/realistic ranges for the endogenously defined prices (which can inter alia exclude zero shadow prices). In fact, such price restrictions have received considerable attention in the DEA literature. For compactness, we will not consider such restrictions in the following illustrative application.
} 
- Inputs: (1) junior research staff (= PhD candidates), (2) senior research staff (= other research personnel). Following Cherchye and Vanden Abeele, we relate the output of each year to the sum of the inputs used in that same year and the inputs used in the two preceding years; this corrects for the fact that output in a particular year may actually result (at least partly) from inputs that have been used in preceding years.

- Outputs: (1) total number of doctoral dissertations, (2) total number of refereed articles in top international journals, and (3) total number of refereed articles in international journals.

The input and output data are taken from the 'Quality Assessment Reports on Research 1996-2000', delivered by each Dutch university in the context of the quinquennial assessment by the VSNU (i.e., the Dutch association of universities). For each research program we have complete data for the years 1998, 1999 and 2000. Pooling the three cross-sections in the same sample, we have 229 observations in total. ${ }^{13}$

Cherchye and Vanden Abeele (2005) provide a detailed discussion about the data and the input-output selection. At this point, two special features of the input-output data deserve some additional explanation. First, the input data account for differences in the allocation of faculty time across different research programs. Specifically, they correct for differences in time spent on teaching in different universities and/or professional ranks (see in particular the discussion on p. 501 in Cherchye and Vanden Abeele). Second, outputs 2 and 3 count publications at the level of the research programs, which effectively avoids double counting publications that are co-authored by researchers of one and the same research program. The particular specification of these outputs (which includes top-journal publications in the output 2 as well as the output 3), entails an implicit extra premium for the top-journal publications. In other terms, it imposes the natural assumption that these publications get a higher weight than other refereed publications in international journals. More specifically, it implies that one input-output combination is a possible comparison partner for another input-output combination only if it produces at least the same amount of articles in international 'top' journals (see the output 2) and, in addition, at least the same amount of articles in refereed journals in general (including top journals; see the output 3). This effectively imposes that a top publication can substitute for another (non-top) publication, but not vice versa. ${ }^{14}$

As a final note, we remark that the testing tools employed below require stricto sensu that the efficiency estimates are independently distributed. This assumption may be criticized as the input values for the years 1998, 1999 and 2000, which are used for computing the efficiency values, are interdependent by construction for each research program. In addition, and probably more importantly, efficiency values are obtained from comparison with a production possibility set that is constructed by means of a common set of reference units; i.e. the observed set of research programs. From that perspective, our (illustrative) test results

\footnotetext{
${ }^{13}$ Recall that the output in a given year is related to the sum of the inputs of that year and those of the two preceding years. Given this, Cherchye and Vanden Abeele use information on 79 research programs. Because of our specific focus on efficiency differences between research programs within specific specialization areas, we restrict attention to the 77 research programs for which the specialization type is known; this leaves 231 observations (= 77 programs x 3 years). From that sample, we further exclude two cases with important missing information, which eventually obtains 229 observations.

${ }^{14}$ Cherchye and Vanden Abeele (2005, p. 501-502) illustrate by means of a simple numerical example. In fact, while our data set implies a two-tiered classification of international journal publications, the same procedure can be used for introducing a three-tiered classification (e.g. 'top journals', 'very good journals' and 'other journals') or any other multi-tiered classification if that would seem recommendable. For the sake of compactness, we abstract from exploring this in our (illustrative) application. Still, we believe that our main qualitative results are fairly robust with respect to such additional journal classifications, which basically imply a more refined output structure.
} 
below should be considered as 'indicative' rather than 'conclusive'. Still, as for our test results based on the full sample of 229 observations (see Table 1), we may refer to the consistency results that have been established for nonparametric efficiency analysis models similar to the one applied here, which suggest that this interdependency problem diminishes for sufficiently large samples (see e.g. Banker, 1993, and Simar and Wilson, 2000). [In this context, it is also worth referring to our discussion in the concluding section, on possible solutions for the sampling problem of DEA analyses.]

\subsection{Overall differences between universities and specialization types: observed prices}

We focus on the necessity condition for cost efficient behavior under economies of scope (see Proposition 2). This necessity condition seems a natural starting point, since inconsistency with this condition implies a fortiori that a $C-R$ of the data set is impossible, and thus that the sufficiency condition can never be met. In this respect, we note that the following results in Tables 1-2 imply that, for each of our exercises, the necessity condition in Proposition 2 is nowhere met at the sample level, which implies redundancy of testing the sufficiency condition in Proposition 3. [Still, while we will not illustrate this in this paper, testing the sufficiency condition is just as simple as testing the necessity condition; recall our discussion in Section 2 on the possibility of using enumeration algorithms.]

For an observation that does not meet the necessary condition for cost efficient behavior, we quantify the degree of cost inefficiency by means of the measure $\varphi$ that was introduced before. Apart from the observed research output and input quantities, this cost efficiency measure also needs input prices. For the different years that we consider, we take the price/wage information from the salary tables that were applicable to the Dutch universities at that time. ${ }^{15}$ One problem in this respect is that salaries depend on the different types of staff (e.g., assistant professor, associate professor, etc.) and seniority. The VSNU-data do not allow us to determine the shares of the different types of research staff that are engaged in a certain university or a research program. Therefore, we assume that all junior researchers have the salary of a third year teaching assistant, while senior researchers are assumed to be of the associate professor level. Note that all price information included in the analysis is in real terms; we constructed real wages on the basis of the Eurostat harmonized consumer price index for the Netherlands. Consistent with our construction of the input quantities (i.e., the output of each year is related to the sum of the input in that same year and the input in the two preceding years) we evaluate the inputs by averaging real wages over the three input years associated with each output year. This yields the following relative input prices (i.e., the ratio of senior staff wage over junior staff wage): 1.745516 for the year $1998,1.745468$ for the year 1999, and 1.745524 for the year 2000. At this point, we note that our above 'simplifications of a complex reality' may lead critics to question the reliability of the prices that we use. Therefore, referring to our earlier discussion of Corollary 1 (and the corresponding linear programming problems in (3.1)), we will use shadow prices in a further exercise, which effectively corrects for potential 'unreliability' of the actual price information.

To begin, we focus on test results for (1) universities (i.e., the corresponding faculties of Economics and Business Management) as a whole and (2) specialization areas as a whole. Both exercises start from efficiency results based upon comparison of each individual research program (in the years 1998, 1999 and 2000) to the full set of 229 research program

\footnotetext{
${ }^{15}$ Dutch universities are subject to a collective agreement that settles working conditions of university personnel. Since 2005, the same collective agreement applies to both public universities and the so-called special universities. Before 2005, there were differences in the agreement for both types of universities. However, the salary settlements were always the same.
} 
observations. ${ }^{16}$ The first exercise then checks (significant) differences in mean efficiency between research programs associated with different universities (while not correcting for different compositions in terms of specialization areas across universities). The second exercise similarly considers (significant) differences in mean efficiency between research programs that are active in different specialization domains (while not correcting for the identity of the organizing university).

Table 1 reports the efficiency results for the newly proposed methodology (in the column 'scope economies' cost efficiency). The table shows mean efficiency values for the different universities and specialization areas in the VSNU-data, respectively; for each (row) category of research programs, the table additionally reports (in the column 'p-value') the probability that the mean efficiency of research programs in that category equals the mean efficiency over all other categories. Focusing on the upper panel of the table, it is clear that there are rather important efficiency differences between the 8 universities. For example, there is a difference of more than 30 percentage points between the two extremes in the sample: Tilburg University obtains a mean efficiency value of about $67.2 \%$, while the University of Nijmegen obtains a mean efficiency of only 35.6\% in the period 1998-2000. [Remark, though, that the efficiency value of the latter university is based upon a rather small sample size.] Overall, the average efficiency level equals $52.1 \%$. The top three universities in terms of mean efficiency values are respectively Tilburg University (67.2\%), Wageningen University (63.3\%) and the Free University of Amsterdam (54.5\%). Note, however, that only three universities perform significantly differently from the average at the $10 \%$ significance level (see the column "pvalue'): Tilburg University and Wageningen University perform significantly better than (the average of) the other universities, while the opposite conclusion applies to the University of Maastricht.

The bottom panel of Table 1 gives the mean efficiency values for the different specialization areas in the sample. Like before, there is considerable efficiency variation over the different specialization areas. In the period 1998-2000, the highest mean efficiency value (of 68.6\%) is obtained in the field of Spatial and Environmental Economics, which is closely followed by Econometrics (mean efficiency of 66.1\%) and Theoretical and Applied Microeconomics (64.8\%). The least efficient areas are Applied Labor Economics and Economics of Public Policy (both areas have a mean efficiency value of $35.3 \%$ ). Five specialization areas performed significantly differently from the mean at the $10 \%$ significance level: Econometrics, Theoretical and Applied Microeconomics, and Spatial and Environmental Economics do significantly better than the rest, while research programs in Applied Labor Economics and Economics of Public Policy do significantly worse.

Based on these results, we may conclude that the average performance of universities may largely be driven by different configurations in terms of specialization domains. Indeed, one interpretation of systematic efficiency differences between research programs that are active in different specialization fields is that alternative specializations entail other research production technologies. To correct for this potential bias in our inter-university comparisons, our following exercises focus on systematic differences between universities per

\footnotetext{
${ }^{16}$ An alternative exercise could have considered a production setting with 30 outputs per university observation, i.e. 3 outputs for each of the 10 specialization areas. However, such a large number of outputs would make the efficiency assessment exercise particularly vulnerable to the so-called 'curse of dimensionality' of nonparametric models, which in this instance would mean a severe upward bias of the efficiency estimates (because we would retain a setting with only 8 university observations for as much as 30 outputs). Therefore, in this study we choose to focus on research programs as production units, and to compute university efficiencies by averaging over the corresponding research programs.
} 
specialization type. ${ }^{17}$ In addition, accounting for possible flaws in our construction of the input prices/wages, we will analyze these differences by using shadow prices.

Before doing so, we briefly compare our results to those obtained on the basis of a standard cost efficiency measure such as that used by Cherchye and Vanden Abeele (2005; see in particular p. 497-499), which does not incorporate the implications of joint input use and input externalities. For each observation $i$, this measure is defined as

$$
\omega^{i}=\frac{\min _{j \in D^{i}}\left(\mathbf{p}^{i}\right)^{\mathbf{x}^{j}}}{\left(\mathbf{p}^{i}\right)^{\prime} \mathbf{x}^{i}} \text { with } D^{i}=\left\{j \in S \mid \mathbf{y}^{j} \geq \mathbf{y}^{i}\right\}
$$

Referring to our discussion in Section 3, we have that $D^{i} \subseteq \mathbf{R}^{i}$ and $\omega^{i} \geq \varphi^{i}$. Thus, our newly proposed method entails a strengthened efficiency analysis. ${ }^{18}$ To interpret this last result in terms of our underlying model of multi-output production, recall from the definition in Section 3 that every output dominating reference set $R^{i}$ in $\mathbf{R}^{i}$ contains a combination of observations $j^{k}$ that each dominate the evaluated observation $i$ in at least one output $k$. The use of the sets $R^{i}$ reflects that combinations of the input vectors $j^{k}$ (in $R^{i}$ ), each producing more of the (individual) outputs $k$ than the evaluated input vector $i$, can also produce the (multioutput) combination $\mathbf{y}^{i}$. As such, our necessity condition for cost efficient behavior under economies of scope naturally complies with the common intuition that such scope economies imply that the cost of the multi-output production should not exceed the sum of the costs associated with the separate production of the individual outputs. It is essentially this feature, which clearly exploits the multiple output production following from scope economies, that entails the strengthened analysis. For example, the standard cost efficiency measure does not consider combined input vectors: the set $D^{i}$ (only) contains observations $j$ that dominate observation $i$ in all outputs simultaneously.

Table 1 reports the results for the measure $\omega^{i}$ in the column 'standard' cost efficiency. Generally, we find that the pattern of the efficiency distribution in that column is similar to that in the column 'scope economies' cost efficiency. For example, we again find that Tilburg University and Wageningen University performed better than the average of the other universities in the period 1998-2000; and we equally obtain that Dutch universities have a comparative advantage in the areas of Spatial and Environmental Economics, Econometrics and Theoretical and Applied Microeconomics. Still, an important observation is that the mean efficiency values obtained by using the newly proposed (scope economies) method are generally lower than those obtained by the standard method. This confirms that an explicit consideration of the features that are specific to multi-output production effectively obtains a more stringent efficiency analysis. In fact, putting an additional a priori structure on the decomposed input vectors or implicit price vectors, which includes specific information regarding the nature of the scope economies (in terms of production externalities and/or

\footnotetext{
${ }^{17}$ Cook et al. (1998) provide a general discussion of issues related to DEA efficiency evaluation when the sample can be subdivided into groups. We note that the procedures they present for dealing with grouped samples in the DEA evaluation could also be used in combination with the methodological tools presented in this paper.

${ }_{18}$ For completeness, we add that Cherchye and Vanden Abeele use shadow prices in their empirical cost efficiency assessment, while the results in Table 1 are based on actual price information. We choose to include the cost efficiency results based on actual price information, as this enhances the comparison with the results in the column 'scope economies' cost efficiency. Still, the main qualitative conclusions of the shadow price assessment are similar to those obtained on the basis of actual prices. The difference is that the cost efficiencies are generally higher (and, thus, the inefficiencies are lower) when using shadow prices rather than actual prices.
} 
jointly used inputs), may entail an even stronger analysis. We briefly return to this in the concluding section.

\section{[Table 1 about here]}

\subsection{Differences between universities per specialization type: shadow prices}

We next decompose the aggregate performance of each specialization area. More specifically, we evaluate each research program by comparing it to all other research programs that are active in the same specialization domain (while -to recall- the results in Table 1 follow from comparison to the set of all research programs independent of their specialization type). Per specialization area, we subsequently calculate the mean efficiency value of each university. This allows us to identify the research domains in which a given university has a comparative advantage as compared to the other universities. This section reports the results of such an exercise when using shadow prices for evaluating the different inputs. ${ }^{19}$ That is, for each observation $i$ we concentrate on the cost efficiency measure $\theta^{i}$ introduced in Section 3.

We allow different shadow prices for different research programs. In doing so, we effectively account for possible salary differences over universities and specialization areas. ${ }^{20}$ Of course, this use of program-specific shadow prices does not account for possible differences in salaries among researchers within one and the same research program; such differences may e.g. follow from different levels of experience, qualifications or productivity. For the current application, it is impossible to account for such differences because we lack the necessary information regarding the composition of the input (= research staff) categories. From that perspective, differences in program-specific shadow prices capture differences in 'average' salaries between research programs (thus reflecting e.g. differences in average experience, qualifications and productivity). At this point, it is also worth recalling the nonuniqueness of the shadow prices computed by means of the linear program in (3.1), whence our following discussion does not focus on the computed shadow prices.

Table 2 tabulates the mean efficiency values of Dutch universities per specialization area. The best performing universities per type of specialization are the following: University of Groningen (Accounting and Finance), Tilburg University (Applied Mathematics; Marketing and Business Economics; Theoretical and Applied Microeconomics), Wageningen University (Development, Growth and Transition), Erasmus University (Econometrics and Applied Labor Economics), University of Maastricht (Econometrics, Applied Labor Economics), Free University of Amsterdam (Applied Labor Economics; Spatial and Environmental Economics), University of Amsterdam (Econometrics; Macroeconomics, Money and International Issues; Economics of Public Policy). In fact, despite the relatively small sample for each specialization domain (ranging from 9 observations to 66 observations), we do find significant efficiency differences for almost all specialization areas (when using the $10 \%$ significance level; see the column 'p-value'). More specifically, we can identify (at the $10 \%$ significance level) universities that do significantly better than the rest (in the fields Marketing and Business Economics; Spatial and Environmental Economics) and, even more

\footnotetext{
${ }^{19}$ We have also conducted similar exercises on the basis of the same (potentially unreliable) price information as before. Generally, this yielded the same qualitative conclusions as for the results in Table 2. Detailed results are available from the authors upon simple request.

${ }^{20}$ Uniform shadow prices across (subgroups of) research programs may be imposed by using the methodological tools that Kuosmanen et al. (2006) proposed in a DEA context; these tools are readily adapted to the current setup.
} 
importantly, universities that perform systematically worse than other institutes (in the fields Accounting and Finance; Applied Mathematics; Development, Growth and Transition; Marketing and Business Economics; Theoretical and Applied Microeconomics; Spatial and Environmental Economics). This indicates that the presented method may obtain robust conclusions even in the case of small samples, when putting minimalistic a priori structure on the multi-output production process and using 'most favorable' shadow prices for evaluating the different research programs. In this specific application setting, such information may be particularly instrumental for robustly benchmarking the bad performing universities: these institutes may learn from other universities which, within the given specialization area, significantly outperform them.

Next, the above list seems to indicate that most universities have a comparative advantage in at least one specialization area. To some extent, these results are in 'contrast' with the information provided by Table 1. Universities that perform well overall (see Table 1) may perform relatively badly in some specialization domains, and universities that perform relatively badly overall may perform well in some specialization areas. For example, Tilburg University, which had the highest mean efficiency value in Table 1, only performs best in the areas of Applied Mathematics, Marketing and Business Economics and Theoretical and Applied Microeconomics. And Wageningen University, which obtained the second highest overall performance value, only excels in the area of Development, Growth and Transition. This indicates that top universities have some 'core' businesses, in which they reach a generally high performance level. Finally, and not surprisingly, universities that generally perform well do not do (significantly) badly in any of the specialization areas that we consider.

In addition, these results seem to confirm our earlier conjecture that one should take into account technology differences between specialization areas in efficiency analyses, which is in contrast with the more naive (but, apparently, rather widespread) view that one may directly compare the performance of research programs that are active in very different specialization areas within the general Economics profession. In fact, disaggregating over specialization domains seems a necessity when assessing the research efficiency of universities: examining aggregate faculty figures, which is conventional practice, does not always provide useful insights in terms of the aim of increasing a university's performance. Quite the contrary: disaggregated figures allow us to situate the comparative advantage of different institutes, which, in turn, can lead to further performance improvements through specialization-specific research policies. As such, this conclusion provides further support for Cherchye and Vanden Abeele's (2005) motivation to focus on micro-units of research production, such as research programs, rather than on macro-units, such as university faculties. From that perspective, the results in Table 2 provide useful complementary information to the results of Cherchye and Vanden Abeele (discussed in the previous section), by specifically considering efficiency differences between universities within one and the same specialization domain. As we indicated above, such results may be useful, for example, from a benchmarking perspective.

\section{[Table 2 about here]}

\section{Summary and concluding remarks}

We have presented a nonparametric methodology for analyzing the cost efficiency of firms that produce multiple outputs. Our starting point is that such multi-output production basically 
refers to economies of scope in the production process, which in turn refers to joint input use and input externalities. Given this, we have instituted a nonparametric characterization of efficient behavior under these general conditions, and subsequently derived necessary and sufficient empirical conditions for data consistency with the cost efficiency requirement. Importantly, these conditions only include observed firm demand and supply data; this means that inputs must not be disaggregated in terms of the specific channels through which they can be allocated (i.e., to a specific output or to joint use for the production of different outputs). Essentially, we have designed cost efficiency conditions that exploit the implications of scope economies (through joint input use and input externalities) at the level of the observable aggregate prices and quantities. In addition, we have relaxed the assumption that input prices are observed, to come up with (linear programming) efficiency tests that utilize shadow prices. Inter alia, this provides a direct link with the nonparametric efficiency assessment literature known as Data Envelopment Analysis (DEA).

We have illustrated our methodology by examining the cost efficient behavior of research programs in Economics and Business Management faculties of Dutch universities. This application shows that the proposed methodology is easy to implement in practice, even for fairly large data sets (e.g., our application involved 229 observations). In fact, we recall from our discussion in Section 4.1 that exploiting scope economies entails a strengthened cost efficiency analysis as compared to more conventionally used alternatives, such as in Cherchye and Vanden Abeele (2005). [As we explain below, the efficiency evaluation can be strengthened further by putting additional structure on the decomposed input vectors and implicit price vectors.] In addition, our application demonstrates the practical usefulness of the method for obtaining robust conclusions regarding cost efficiency differences between universities within specific specialization areas, also when using shadow prices to evaluate the different inputs. As we have indicated, in our specific application set-up such insights may be particularly useful for benchmarking purposes.

A general qualitative conclusion of our results is that they seem to support the necessity of accounting for technological differences between specialization domains (within the general Economics profession) when analyzing research performance. Given this, we have analyzed performance differences between universities at the level of individual specialization areas. We found that universities indeed seem to specialize in only a few research domains: while universities that perform best overall generally perform well in all the domains in which they are active, universities that are generally less efficient can also perform very well in certain areas of specialization. The fact that many of these findings turned out to be statistically significant illustrates that our method can obtain robust conclusions while imposing a minimalistic a priori structure on the actual (but unknown) production process (even in the cases where we could only use small (specialization-specific) data sets).

At this point, it is worth stressing the limitations of our empirical analysis, which mainly served to illustrate the proposed methodology. Most importantly, we did not explicitly account for errors-in-variables and small sample bias. Still, we want to indicate that (i) we have used an input-output configuration that largely coincides with that considered by the VSNU in their quinquennial assessment based on the same data, and (ii) the data, which were reported by the universities themselves in extensive self-assessments, are relatively well standardized and have been subject to some scrutiny for correctness and consistency, which gives us reasonable confidence in their quality. But in order to draw absolute conclusions from our exercises, it seems recommendable to utilize methodological tools that satisfactorily deal with errors-in-variables. See, for example, Grosskopf (1996) for a survey of tools that are currently available in the nonparametric literature, and Cazals et al. (2002) for a more recent proposal; these tools (that were originally proposed in a DEA context) may easily be accommodated to the newly proposed cost efficiency assessment methodology. Next, the 
sampling problem may apply in particular to our specialization-specific exercises, which are often based on a fairly limited number of observations. To obtain more robust results in such cases, one may use the bootstrap procedure proposed (again, in a DEA context) by Simar and Wilson (1998), which is also readily adapted to the presented efficiency evaluation tools.

Three concluding remarks are in order. First, to keep the discussion focused, we have concentrated on consistency testing and the associated efficiency measurement. Still, Varian (1984) emphasized alternative uses of the nonparametric approach in addition to testing for optimizing firm behavior, namely recovering the production set and forecasting firm behavior under new price conditions. We emphasize that such recoverability and forecasting questions may also be addressed when starting from the specific (scope economies-based) condition for cost efficiency that has been forwarded in this study; the methodological extensions develop along directly analogous lines as in Varian (1984). The goodness-of-fit and subset rationalization concepts of, respectively, Varian (1990) and Banker and Maindiratta (1988) allow for addressing such questions while accounting for observed inefficiencies.

Another remark pertains to the shadow price efficiency measurement problem in (3.1), which is applicable when reliable price information is not available. Such a shadow price analysis can be strengthened by imposing price information in the form of additional constraints that define a feasible range for the relative prices, which may rule out the extreme cases where the relative price of a commodity approaches zero or infinity. The technical questions related to incorporating such price restrictions have been discussed extensively in a DEA context, most commonly under the label 'weight restrictions' or 'assurance regions' (see, e.g., Allen et al., 1997; Pedraja-Chaparro et al., 1997, for surveys; and Kuosmanen et al., 2006, for more recent developments). These tools are readily adapted to the current set-up.

Finally, from a related perspective, putting an additional a priori structure on the decomposed input vectors and the implicit price vectors will obviously strengthen the cost efficiency analysis of the multi-output production process at hand. Extra structure on the decomposed input vectors may, for example, reflect additional information (or assumptions) regarding the presence of jointly used inputs, or regarding (shares of) inputs that are specifically used for the production of particular outputs; similarly, additional structure on the implicit price vectors may reflect some a priori position regarding the presence of externalities in the production of certain outputs. Generally, such extra price-quantity conditions will entail refinements of the general model presented in Section 2, which in turn will lead to more stringent necessary and sufficient cost efficiency conditions in terms of observables (see Section 3). [These conditions may be obtained along similar lines as in the proofs of Propositions 2 and 3.].

In this respect, it is also interesting to compare our approach with Activity Based Costing (ABC) (e.g., Cooper and Kaplan, 1988; Christensen and Demski, 1995, elaborate on the relationship between $\mathrm{ABC}$ and the classical theory of cost). Essentially, $\mathrm{ABC}$ uses information regarding the input costs (= prices $\mathrm{x}$ quantities) that are (through so-called 'cost drivers') allocated to individual outputs. By contrast, our approach does not require such information, but starts from a separate allocation of input quantities (resulting in decomposed input vectors) and input prices (resulting in implicit price vectors) to different outputs. From this perspective, $\mathrm{ABC}$ can be considered complementary to our approach: the input cost information used in $\mathrm{ABC}$ can be useful for putting a priori restrictions on the feasible combinations of decomposed input vectors and implicit price vectors.

In summary, the approach to modeling multi-output production presented here provides a general framework that encompasses a wide spectrum of production models that incorporate input externalities and joint input use. 


\section{Appendix}

\section{A. Proof of Proposition 1}

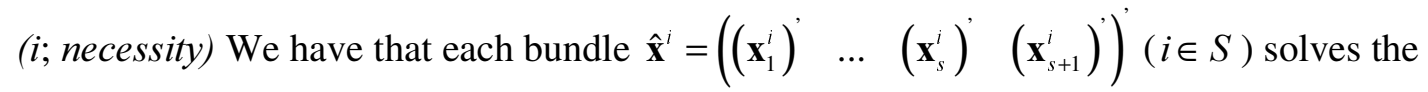
following problem for given priority weight vector $\boldsymbol{\mu}^{i}:^{21}$

$$
\max _{\mathbf{x}_{1}^{i}, \ldots, \mathbf{x}_{s}^{i}, \mathbf{x}_{s+1}^{i}} \sum_{k \in K} \mu_{k}^{i} f_{k}\left(\mathbf{x}_{1}^{i}, \ldots, \mathbf{x}_{s+1}^{i}\right) \quad \text { s.t. } \quad\left(\mathbf{p}^{i}\right)^{\prime}\left(\mathbf{x}_{1}^{i}+\ldots+\mathbf{x}_{s+1}^{i}\right) \leq\left(\mathbf{p}^{i}\right)^{\prime} \mathbf{x}^{i}
$$

Given concavity, the output-specific production functions are subdifferentiable, which carries over to their weighted $\operatorname{sum} \sum_{k \in K} \mu_{k}^{i} f_{k}$. An optimal solution to the above maximization problem should therefore satisfy (for $l=1, \ldots, s+1$ )

$$
\sum_{k \in K} \mu_{k}^{i} f_{k}^{\mathbf{x}^{i}} \leq \eta^{i} \mathbf{p}^{i}
$$

for $\eta^{i}$ the Lagrange multiplier associated with the cost constraint, and $f_{k}^{\mathbf{x}^{l}}$ a subgradient of the production function $f_{k}$ defined for the vector $\mathbf{x}^{l}$ and evaluated at

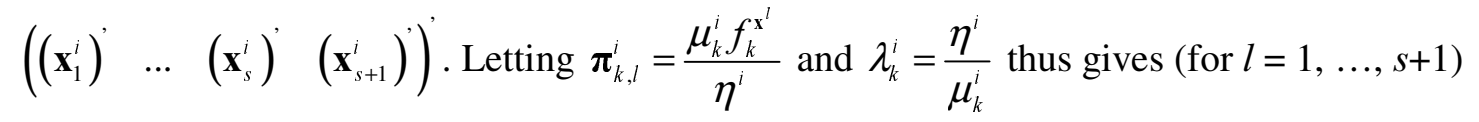

$$
f_{k}^{\mathbf{x}^{l}} \leq \lambda_{k}^{i} \boldsymbol{\pi}_{k, l}^{i}
$$

Next, concavity of the functions $f_{k}$ implies for all $k \in K$

$$
f_{k}\left(\mathbf{x}_{1}^{j}, \ldots, \mathbf{x}_{s+1}^{j}\right)-f_{k}\left(\mathbf{x}_{1}^{i}, \ldots, \mathbf{x}_{s+1}^{i}\right) \leq \sum_{l=1, \ldots, s+1}\left(f_{k}^{\mathbf{x}^{l}}\right)^{\prime}\left(\mathbf{x}_{l}^{j}-\mathbf{x}_{l}^{i}\right)
$$

Substituting (A.1) in (A.2) and setting $y_{k}^{i}=f_{k}\left(\mathbf{x}_{1}^{i}, \ldots, \mathbf{x}_{s+1}^{i}\right)$ obtains

$$
y_{k}^{j}-y_{k}^{i} \leq \lambda_{k}^{i} \sum_{l=1, \ldots, s+1}\left(\boldsymbol{\pi}_{k, l}^{i}\right)^{\prime}\left(\mathbf{x}_{l}^{j}-\mathbf{x}_{l}^{i}\right),
$$

which is equivalent to

$$
y_{k}^{j} \geq y_{k}^{i} \Rightarrow \sum_{l=1, \ldots, s+1}\left(\boldsymbol{\pi}_{k, l}^{i}\right)^{\prime} \mathbf{x}_{l}^{j} \geq \sum_{l=1, \ldots, s+1}\left(\boldsymbol{\pi}_{k, l}^{i}\right)^{\prime} \mathbf{x}_{l}^{i} .
$$

\footnotetext{
${ }^{21}$ To be exact, we have that $f_{k}$ is quasi-concave rather than concave. For compactness, however, we consider $f_{k}$ concave in the following proof. Indeed, under mild regularity conditions, for any quasi-concave function there exists a positive monotone transformation that obtains a concave representative. Such a monotone transformation is harmless in view of the equivalence between (A.3) and (A.4), which applies to any monotonous transformation of $f_{k}$.
} 
(ii; sufficiency) Recalling the equivalence between (A.3) and (A.4), we first define (for $k=1$, ..., s)

$$
f_{k}\left(\mathbf{x}_{1}, \ldots, \mathbf{x}_{s+1}\right)=\min _{i \in S}\left[y_{k}^{i}+\lambda_{k}^{i} \sum_{l=1, \ldots, s+1}\left(\boldsymbol{\pi}_{k, l}^{i}\right)^{\prime}\left(\mathbf{x}_{l}-\mathbf{x}_{l}^{i}\right)\right]
$$

Varian (1984, Theorem 2) proves that $f_{k}\left(\mathbf{x}_{1}, \ldots, \mathbf{x}_{s+1}\right)=y_{k}^{i}$. Next, given $\boldsymbol{\mu}^{i} \in \mathfrak{R}_{+}^{s}$, we have for all $\hat{\mathbf{x}}$ such that $\left(\mathbf{p}^{i}\right)^{\prime}\left(\sum_{l=1}^{s+1} \mathbf{x}_{l}\right) \leq\left(\mathbf{p}^{i}\right)^{\prime} \mathbf{x}^{i}$

$$
\sum_{k \in K} \mu_{k}^{i} f_{k}\left(\mathbf{x}_{1}, \ldots, \mathbf{x}_{s+1}\right) \leq \sum_{k \in K} \mu_{k}^{i}\left[y_{k}^{i}+\lambda_{k}^{i} \sum_{l=1, \ldots, s+1}\left(\boldsymbol{\pi}_{k, l}^{i}\right)^{\prime}\left(\mathbf{x}_{l}-\mathbf{x}_{l}^{i}\right)\right]
$$

Without losing generality, we concentrate on $\mu_{k}^{i}=\left(\lambda_{1}^{i} / \lambda_{k}^{i}\right)$, which obtains

$$
\sum_{k \in K} \mu_{k}^{i} f_{k}\left(\mathbf{x}_{1}, \ldots, \mathbf{x}_{s+1}\right) \leq \sum_{k \in K} \mu_{k}^{i} y_{k}^{i}+\lambda_{1}^{i}\left[\sum_{l=1, \ldots, s+1}\left(\mathbf{p}^{i}\right)^{\prime}\left(\mathbf{x}_{l}-\mathbf{x}_{l}^{i}\right)\right]
$$

Since $\left(\mathbf{p}^{i}\right)^{\prime}\left(\sum_{l=1}^{s+1} \mathbf{x}_{l}\right) \leq\left(\mathbf{p}^{i}\right)^{\prime} \mathbf{x}^{i}$, we thus have

$$
\sum_{k \in K} \mu_{k}^{i} f_{k}\left(\mathbf{x}_{1}, \ldots, \mathbf{x}_{s+1}\right) \leq \sum_{k \in K} \mu_{k}^{i} y_{k}^{i}=\sum_{k \in K} \mu_{k}^{i} f_{k}\left(\mathbf{x}_{1}^{i}, \ldots, \mathbf{x}_{s+1}^{i}\right)
$$

which proves that $\hat{\mathbf{x}}^{i}$ maximizes $\sum_{k \in K} \mu_{k}^{i} f_{k}\left(\mathbf{x}_{1}, \ldots, \mathbf{x}_{s+1}\right)$ subject to $\left(\mathbf{p}^{i}\right)^{\prime}\left(\sum_{l=1}^{s+1} \mathbf{x}_{l}\right) \leq\left(\mathbf{p}^{i}\right)^{\prime} \mathbf{x}^{i}$. We conclude that the functions $f_{k}\left(\mathbf{x}_{1}, \ldots, \mathbf{x}_{s+1}\right)(k \in K)$ in (A.5) provide a $C-R$ of the data. These functions are concave, monotonously increasing and continuous (see Varian, 1984, Theorem 2). The corresponding input requirement sets are closed, convex and positive monotonous.

\section{B. Proof of Proposition 2}

For any set $R^{i}$ with $\forall k \in K: \exists j^{k} \in R^{i}: y_{k}^{j^{k}} \geq y_{k}^{i}$ consistency with the $C$ - $R$ conditions in Proposition 1 requires $\forall k: y_{k}^{j^{k}} \geq y_{k}^{i} \Rightarrow \sum_{l=1, \ldots, s+1}\left(\boldsymbol{\pi}_{k, l}^{i}\right)^{\prime} \mathbf{x}_{l}^{j^{k}} \geq \sum_{l=1, \ldots, s+1}\left(\boldsymbol{\pi}_{k, l}^{i}\right)^{\prime} \mathbf{x}_{l}^{i} \quad$ and thus $\sum_{k \in K}\left(\sum_{l=1, \ldots, s+1}\left(\boldsymbol{\pi}_{k, l}^{i}\right)^{\prime} \mathbf{x}_{l}^{j^{k}}\right) \geq \sum_{k \in K}\left(\sum_{l=1, \ldots, s+1}\left(\boldsymbol{\pi}_{k, l}^{i}\right)^{\prime} \mathbf{x}_{l}^{i}\right)$. The result consequently follows from the definitional fact that $\quad \sum_{j^{k} \in R^{i}}\left(\mathbf{p}^{i}\right)^{\mathbf{x}^{j^{k}}} \geq \sum_{k \in K}\left(\sum_{l=1, \ldots, s+1}\left(\boldsymbol{\pi}_{k, l}^{i}\right)^{\prime} \mathbf{x}_{l}^{j^{k}}\right) \quad$ and $\sum_{k \in K}\left(\sum_{l=1, \ldots, s+1}\left(\boldsymbol{\pi}_{k, l}^{i}\right)^{\prime} \mathbf{x}_{l}^{i}\right)=\left(\mathbf{p}^{i}\right)^{\prime} \mathbf{x}^{i}$. 


\section{Proof of Proposition 3}

Suppose that a construction $S_{k}(k \in K)$ consistent with the sufficiency condition in Proposition 3 exists. Given this, we can construct a configuration of decomposed input vectors and implicit price vectors that meet the $C-R$ conditions in Proposition 1 . Specifically, we use

$$
\begin{aligned}
& \text { for } i \in S: \boldsymbol{\pi}_{k_{1}, k_{2}}^{i}=\mathbf{p}^{i} \text { for } k_{1}=k_{2}, \boldsymbol{\pi}_{k_{1}, k_{2}}^{i}=\mathbf{0} \text { for } k_{1} \neq k_{2} ; \text { and } \\
& \text { for } i \in S_{k}: \mathbf{x}_{k}^{i}=\mathbf{x}^{i} \text {. }
\end{aligned}
$$

We obtain the sufficiency result in two steps. First, for $i \in S_{k}$ we have for any $j \in S$ : if $y_{k}^{j} \geq y_{k}^{i}$, then $j \in S_{k}$. (Indeed, $j \in S_{k^{*}}, k^{*} \neq k$ would require $y_{k}^{j}<y_{k}^{i}$, which is not the case.) Given this, we can distinguish two cases for each $i, j \in S$ with $y_{k}^{j} \geq y_{k}^{i}$. If $i \in S_{k}$ then $j \in S_{k}$

(see before) and consequently $\left(\mathbf{p}^{i}\right)^{\prime} \mathbf{x}^{i}=\sum_{l=1, \ldots, s+1}\left(\boldsymbol{\pi}_{k, l}^{i}\right)^{\prime} \mathbf{x}_{l}^{i} \leq \sum_{l=1, \ldots, s+1}\left(\boldsymbol{\pi}_{k, l}^{i}\right)^{\prime} \mathbf{x}_{l}^{j}=\left(\mathbf{p}^{i}\right)^{\prime} \mathbf{x}^{j} \quad$ by construction. Alternatively, if $i \notin S_{k}$ we have $\sum_{l=1, \ldots, s+1}\left(\boldsymbol{\pi}_{k, l}^{i}\right) \mathbf{x}_{l}^{i}=0$ and the condition $0=\sum_{l=1, \ldots, s+1}\left(\boldsymbol{\pi}_{k, l}^{i}\right)^{\prime} \mathbf{x}_{l}^{i} \leq \sum_{l=1, \ldots, s+1}\left(\boldsymbol{\pi}_{k, l}^{i}\right)^{\prime} \mathbf{x}_{l}^{j}$ is always satisfied.

\section{References}

Afriat, S. (1972), Efficiency Estimation of Production Functions, International Economic Review 13, 568-598.

Allen, R., A.D. Athanassopoulos, R.G. Dyson, and E. Thanassoulis (1997), Weight Restrictions and Value Judgements in DEA: Evolution, Development and Future Directions, Annals of Operations Research 73, 13-34.

Banker R. (1993), Maximum Likelihood, Consistency and Data Envelopment Analysis: A Statistical Foundation, Management Science 39, 1265-1273.

Banker, R.D. and A Maindiratta (1988), Nonparametric Analysis of Technical and Allocative Efficiencies in Production, Econometrica 56, 1315-1332.

Baumol, W., J. Panzar and R. Willig (1982), Contestable Markets and the Theory of Industry Structure, Harcourt Brace Jovanovich, New York.

Bogetoft, P. (1996), DEA on Relaxed Convexity Assumptions, Management Science 42, 457-465.

Cazals S., J-P. Florens and L. Simar (2002), Nonparametric Frontier Estimation: A Robust Approach, Journal of Econometrics 106, 1-25.

Charnes, A., W.W. Cooper and E. Rhodes (1978), Measuring the Efficiency of Decision Making Units, European Journal of Operational Research 2, 429-444.

Cherchye, L., B. De Rock and F. Vermeulen (2004), The Collective Model of Household Consumption: a Nonparametric Characterization, CentER Discussion Paper, 2004-76, CentER, Tilburg University.

Cherchye, L. and P. Vanden Abeele (2005), On Research Efficiency: A Micro-Analaysis of Dutch University Research in Economics and Business Management, Research Policy 34, 495-516.

Christensen, J. and J.S. Demski (1995), The Classical Foundations of 'Modern' Costing, Management Accounting Research 6, 13-32.

Cook, W.D., D. Chai, J. Doyle and R. Green (1998), Hierarchies and Groups in DEA, Journal of Productivity Analysis 10, 177-198. 
Cooper R. and Kaplan R. (1988), Measure Costs Right: Make the Right Decisions, Harvard Business Review, sep-oct 1988, 96-103.

Cooper, W.W., L.M. Seiford and K. Tone (2000), Data Envelopment Analysis: A Comprehensive Text with Models, Applications, References and DEA-Solver Software, Kluwer Academic Publishers, Dordrecht.

Färe, R. and S. Grosskopf (1995), Non-Parametric Tests of Regularity, Farrell Efficiency, and Goodness of Fit, Journal of Econometrics 69, 415-425.

Färe, R., S. Grosskopf and C.A.K. Lovell (1994), Production Frontiers, Cambridge University Press, Cambridge.

Farrell, M.J. (1957), The Measurement of Productive Efficiency, Journal of the Royal Statistical Society Series A 120, 253-281.

Grosskopf, S. (1996), Statistical Inference and Non-Parametric Efficiency: A Selective Survey, Journal of Productivity Analysis 7, 161-176.

Hanoch, G., and M. Rothschild (1972), Testing Assumptions of Production Theory: A Nonparametric Approach, Journal of Political Economy 80, 256-275.

Kim, K., J.-P. Chavas, J. Foltz and B. Barham (2005), Research and Development at U.S. Research Universities: An analysis of Scope Economies, Mimeo, Department of Agricultural Economics \& Rural Development, Seoul National University.

Kuosmanen, T., L. Cherchye and T. Sipiläinen (2006), The Law of one Price in Data Envelopment Analysis: Restricting Weight Flexibility across Firms, European Journal of Operational Research 170, 735-757.

Mas-Colell, A., M. Whinston and J. Green (1995), Microeconomic Theory, Oxford University Press, Oxford.

Myles, G.D. (1995), Public Economics, Cambridge University Press, Cambridge.

Pedraja-Chaparro, F., J. Salinas-Jimenez, and P. Smith (1997), On the Role of Weight Restrictions in Data Envelopment Analysis, Journal of Productivity Analysis 8, 215-230.

Petersen, N.C. (1990), Data Envelopment Analysis on a Relaxed Set of Assumptions, Management Science 36, 305-314.

Simar, L. and P. Wilson (1998), Sensitivity Analysis of Efficiency Scores: How to Bootstrap in Nonparametric Frontier Models, Management Science 44, 49-61.

Varian, H.R. (1984), The Non-Parametric Approach to Production Analysis, Econometrica 52, 579598.

Varian, H.R. (1990), Goodness-of-Fit in Optimizing Models, Journal of Econometrics 46, 125-140.

Yatchew, A. (1998), Nonparametric Regression Techniques in Economics, Journal of Economic Literature 36, 669-721. 
Table 1: Differences between universities and specialization types

\begin{tabular}{|l|c:ccccc|}
\hline & & 'scope economies' cost efficiency & 'standard' cost efficiency \\
\hline & number & mean efficiency & st. dev. & p-value & \multicolumn{2}{c|}{$\begin{array}{c}\text { mean } \\
\text { sfficiency }\end{array}$} \\
\hline University & & & & & & \\
Erasmus University of Rotterdam & 59 & 0.528 & 0.303 & 0.831 & 0.562 & 0.320 \\
Tilburg University & 27 & 0.672 & 0.273 & 0.004 & 0.741 & 0.282 \\
University of Nijmegen & 6 & 0.356 & 0.173 & 0.156 & 0.423 & 0.252 \\
University of Groningen & 18 & 0.437 & 0.213 & 0.197 & 0.537 & 0.275 \\
University of Maastricht & 27 & 0.417 & 0.214 & 0.046 & 0.469 & 0.283 \\
University of Amsterdam & 35 & 0.454 & 0.284 & 0.136 & 0.466 & 0.294 \\
Free University of Amsterdam & 36 & 0.545 & 0.324 & 0.597 & 0.566 & 0.331 \\
Wageningen University & 21 & 0.633 & 0.282 & 0.063 & 0.662 & 0.300 \\
Overall & 229 & 0.521 & 0.289 & & 0.562 & 0.311 \\
\hline Specialization area & & & & & & \\
accounting and finance & 36 & 0.467 & 0.280 & 0.224 & 0.513 & 0.309 \\
applied mathematics & 18 & 0.514 & 0.284 & 0.917 & 0.529 & 0.299 \\
development, growth and transition & 15 & 0.485 & 0.279 & 0.611 & 0.548 & 0.317 \\
econometrics & 15 & 0.661 & 0.214 & 0.053 & 0.733 & 0.229 \\
applied labor economics & 13 & 0.353 & 0.155 & 0.030 & 0.384 & 0.205 \\
marketing and business economics & 66 & 0.493 & 0.291 & 0.345 & 0.539 & 0.315 \\
macroeconomics, money and international issues & 18 & 0.547 & 0.279 & 0.693 & 0.591 & 0.299 \\
theoretical and applied microeconomics & 21 & 0.648 & 0.286 & 0.034 & 0.692 & 0.310 \\
economics of public policy & 9 & 0.353 & 0.219 & 0.075 & 0.354 & 0.218 \\
spatial and environmental economics & 18 & 0.686 & 0.352 & 0.011 & 0.696 & 0.358 \\
Overall & 229 & 0.521 & 0.289 & & 0.562 & 0.311 \\
\hline
\end{tabular}

Note: The column p-value reports the (two-sided) probability value for the hypothesis that the mean efficiency of the row categories equals the mean efficiency over all other categories. 
Table 2: Differences between universities per specialization type; efficiency differences based on shadow prices

\begin{tabular}{|c|c|c|c|c|}
\hline \multirow{2}{*}{\multicolumn{5}{|c|}{\begin{tabular}{|l} 
Accounting and finance \\
\end{tabular}}} \\
\hline & & & & \\
\hline Erasmus University of Rotterdam & 6 & 0.826 & 0.357 & 0.481 \\
\hline Tilburg University & 6 & 0.831 & 0.171 & 0.457 \\
\hline University of Groningen & 3 & 1.000 & 0.000 & 0.146 \\
\hline University of Maastricht & 6 & 0.722 & 0.309 & 0.886 \\
\hline University of Amsterdam & 9 & 0.576 & 0.365 & 0.078 \\
\hline Free University of Amsterdam & 6 & 0.696 & 0.385 & 0.720 \\
\hline Overall & 36 & 0.740 & 0.322 & \\
\hline \multicolumn{5}{|l|}{ Applied mathematics } \\
\hline Erasmus University of Rotterdam & 9 & 0.787 & 0.300 & 0.080 \\
\hline Tilburg University & 3 & 1.000 & 0.000 & 0.344 \\
\hline University of Amsterdam & 3 & 0.970 & 0.052 & 0.482 \\
\hline Free University of Amsterdam & 3 & 0.959 & 0.071 & 0.541 \\
\hline Overall & 18 & 0.881 & 0.230 & \\
\hline \multicolumn{5}{|l|}{ Development, growth and transition } \\
\hline University of Groningen & 3 & 0.999 & 0.001 & 0.293 \\
\hline University of Maastricht & 3 & 0.348 & 0.070 & 0.000 \\
\hline Free University of Amsterdam & 6 & 0.950 & 0.088 & 0.249 \\
\hline Wageningen University & 3 & 1.000 & 0.000 & 0.291 \\
\hline Overall & 15 & 0.849 & 0.267 & \\
\hline \multicolumn{5}{|l|}{ Econometrics } \\
\hline Erasmus University of Rotterdam & 3 & 1.000 & 0.000 & 0.460 \\
\hline Tilburg University & 3 & 0.958 & 0.073 & 0.117 \\
\hline University of Maastricht & 3 & 1.000 & 0.000 & 0.460 \\
\hline University of Amsterdam & 3 & 1.000 & 0.000 & 0.460 \\
\hline Free University of Amsterdam & 3 & 0.973 & 0.028 & 0.481 \\
\hline Overall & 15 & 0.986 & 0.035 & \\
\hline \multicolumn{5}{|l|}{ Applied labor economics } \\
\hline Erasmus University of Rotterdam & 2 & 1.000 & 0.000 & 0.689 \\
\hline University of Maastricht & 3 & 1.000 & 0.000 & 0.606 \\
\hline University of Amsterdam & 5 & 0.982 & 0.040 & 0.220 \\
\hline Free University of Amsterdam & 3 & 1.000 & 0.000 & 0.606 \\
\hline Overall & 13 & 0.993 & 0.025 & \\
\hline \multicolumn{5}{|l|}{ Marketing and business economics } \\
\hline Erasmus University of Rotterdam & 18 & 0.766 & 0.238 & 0.483 \\
\hline Tilburg University & 9 & 0.905 & 0.158 & 0.033 \\
\hline University of Nijmegen & 3 & 0.544 & 0.228 & 0.228 \\
\hline University of Groningen & 12 & 0.589 & 0.271 & 0.047 \\
\hline University of Maastricht & 9 & 0.713 & 0.268 & 0.857 \\
\hline University of Amsterdam & 3 & 0.474 & 0.124 & 0.095 \\
\hline Free University of Amsterdam & 6 & 0.715 & 0.390 & 0.904 \\
\hline Wageningen University & 6 & 0.881 & 0.243 & 0.145 \\
\hline Overall & 66 & 0.728 & 0.269 & \\
\hline
\end{tabular}




\begin{tabular}{|l|c:ccc|}
\hline Macroeconomics, money and international issues & & & & \\
Erasmus University of Rotterdam & 6 & 0.942 & 0.102 & 0.188 \\
Tilburg University & 3 & 0.998 & 0.004 & 0.442 \\
University of Nijmegen & 3 & 0.995 & 0.009 & 0.498 \\
University of Maastricht & 3 & 0.948 & 0.051 & 0.521 \\
University of Amsterdam & 3 & 1.000 & 0.000 & 0.405 \\
Overall & 18 & 0.971 & 0.064 & \\
\hline Theoretical and applied microeconomics & & & & \\
Erasmus University of Rotterdam & 6 & 0.808 & 0.298 & 0.612 \\
Tilburg University & 3 & 1.000 & 0.000 & 0.199 \\
University of Amsterdam & 3 & 0.607 & 0.198 & 0.036 \\
Free University of Amsterdam & 3 & 0.966 & 0.059 & 0.324 \\
Wageningen University & 6 & 0.872 & 0.159 & 0.755 \\
Overall & 21 & 0.848 & 0.219 & \\
\hline Economics of public policy & & & & \\
Erasmus University of Rotterdam & 6 & 0.948 & 0.124 & 0.506 \\
University of Amsterdam & 3 & 1.000 & 0.000 & 0.506 \\
Overall & 9 & 0.965 & 0.102 & \\
\hline Spatial and environmental economics & & & & \\
\hline Erasmus University of Rotterdam & 3 & 0.401 & 0.213 & 0.000 \\
University of Amsterdam & 3 & 0.928 & 0.125 & 0.609 \\
Free University of Amsterdam & 6 & 1.000 & 0.000 & 0.087 \\
Wageningen University & 6 & 0.907 & 0.185 & 0.566 \\
Overall & 18 & 0.857 & 0.251 & \\
\hline
\end{tabular}

Note: The column p-value reports, for each specialization type, the (two-sided) probability value for the hypothesis that the mean efficiency of the row categories equals the mean efficiency over all other categories. 